\title{
Assessment of the basic parameters of the CERN Superconducting Proton Linac
}

\author{
O. Brunner, ${ }^{1}$ S. Calatroni, ${ }^{1}$ E. Ciapala, ${ }^{1}$ M. Eshraqi,${ }^{1,2}$ R. Garoby, ${ }^{1}$ F. Gerigk, ${ }^{1}$ A. Lombardi, ${ }^{1}$ R. Losito, ${ }^{1}$ V. Parma, ${ }^{1}$ \\ C. Rossi, ${ }^{1}$ J. Tuckmantel, ${ }^{1}$ M. Vretenar, ${ }^{1}$ U. Wagner, ${ }^{1}$ and W. Weingarten ${ }^{1}$ \\ ${ }^{1}$ CERN, Geneva, Switzerland \\ ${ }^{2}$ School of Accelerators and Particles, IPM (Institute for Research in Fundamental Sciences) P.O. Box 19395-5531, Tehran, Iran
} (Received 3 December 2008; published 14 July 2009)

\begin{abstract}
The construction of a $4 \mathrm{GeV}$ superconducting proton linac (the SPL) is now part of the long term plan of CERN, and the construction of Linac4, its low-energy front end, has begun. For mid-2012 the existing conceptual design of the SPL has to be refined and transformed into a project proposal. As a first step, basic parameters like rf frequency, accelerating gradient, and operating temperature of the superconducting cavities have been reassessed, taking into account the experience accumulated in the world during the recent years, especially for the Spallation Neutron Source (SNS) in Oakridge and the International Linear Collider (ILC) projects. The conclusions confirm the validity of the initial choices, namely, the rf frequency of $704.4 \mathrm{MHz}$ and the cooling temperature of $\approx 2 \mathrm{~K}$. However, the assumed gradients are estimated as optimistic: additional tests are necessary during the coming years to properly define the values to be used in the SPL design. This analysis is documented and its results are explained in this report.
\end{abstract}

DOI: $10.1103 /$ PhysRevSTAB.12.070402

PACS numbers: 29.20.Ej

\section{INTRODUCTION}

The first proposal for building a superconductor proton linac (SPL) at CERN to replace some of the existing accelerators was made 12 years ago [1]. It was soon realized that such an accelerator could evolve towards very high beam power, which would allow for new physics facilities concerning neutrinos and/or radioactive ion beams [2]. These first versions of the design were using exclusively the superconducting cavities and the rf equipment available after the decommissioning of the Large Electron Positron collider (LEP) at CERN. The rf frequency was therefore the same all along the accelerator (352.2 MHz), and the peak beam power (beam current during the pulse $\times$ kinetic energy) was limited by the number of klystrons on hand.

These constraints were later considered as excessive, as the LEP equipment was aging/degrading and the characteristics of state-of-the-art superconducting cavities were progressing far beyond the ones of LEP. The low-energy part of the SPL (up to $160 \mathrm{MeV}$ ), was not concerned because $352.2 \mathrm{MHz}$ is a quasioptimum frequency for the acceleration of protons in this energy range. On the other hand, the high-energy part was redesigned using a different rf frequency and hence new cavities and better matched pulsed klystrons. A frequency of $704.4 \mathrm{MHz}$ was selected in the conceptual design published in 2006 [3], which allowed one to increase the beam energy to $3.5 \mathrm{GeV}$ and the current during the beam pulse to $40 \mathrm{~mA}$, while reducing the accelerator length from 800 to $430 \mathrm{~m}$.

In 2007 the construction of new injectors to replace the existing Linac2, PSB (PS booster) and PS (proton synchrotron), was declared as part of the long term plan of CERN [4]. That consists of a low-power $4 \mathrm{GeV}$ version of the SPL
(LP-SPL) [5] and a new $50 \mathrm{GeV}$ slow-cycling synchrotron (PS2) [6]. Hence, the construction of Linac4 [7], the lowenergy front-end of the SPL, has started in 2008 in view of replacing Linac2 in 2014, and the detailed studies of the superconducting part of the SPL and of PS2 have begun at the same time, with the aim of submitting project proposals by mid-2012. In that context, the basic parameters of the SPL have been reassessed during the first half of 2008, to optimize cost and performance and to make the best use of the extensive experience accumulated around the world especially at $800 \mathrm{MHz}$ for the SNS and at $1.3 \mathrm{GHz}$ for the ILC. That was the subject of a special review meeting April 2008 [8]).

This report is based on the work done by the participants before the meeting as well as after, taking into account the comments and reactions that it triggered. After listing the requirements (Sec. II), the linac architectures and the different $\mathrm{rf}$ frequencies that have been considered are described and commented on in Sec. III. The potential performance of superconducting cavities and their dependence on frequency and cooling temperature are analyzed in Sec. IV, as well as the impact on electrical power consumption. The dependence of basic electromagnetic characteristics of $\mathrm{rf}$ cavities as a function of frequency and number of cells is recapitulated in Sec. V. Cryogenics considerations are treated in Sec. VI. The $\mathrm{rf}$ hardware issues are the subject of Sec. VII. Conclusions are drawn in Sec. VIII.

\section{BEAM SPECIFICATIONS}

\section{A. Introduction}

The design of the SPL is meant to fulfill two main purposes: (i) It shall provide a suitable $\mathrm{H}^{-}$beam for 
TABLE I. PS2 parameters.

\begin{tabular}{lcc}
\hline \hline Parameter & Value & Comment \\
\hline Injection energy & $4 \mathrm{GeV}$ & Space charge PS2 \\
Extraction energy & $50 \mathrm{GeV}$ & $2 \times$ PS, SPS improvement \\
Circumference & $1346.4 \mathrm{~m}$ & PS2/SPS size ratio: $15 / 77$ \\
Cycling period & $2.4 \mathrm{~s}$ & \\
Harmonic number (25/50/75 ns bunch spacing) & $180 / 90 / 60$ & $10 \%$ losses in PS2 \\
Protons per LHC bunch/cycle & $4.0 \times 10^{11} / 6.72 \times 10^{13}$ & Twice the ultimate brightness $+10 \%$ for losses \\
$(25 \mathrm{~ns}$ bunch spacing) at ejection & & Fixed target physics \\
Protons per LHC bunch/cycle & $4.4 \times 10^{11} / 7.39 \times 10^{13}$ & \\
$(25 \mathrm{~ns}$ bunch spacing) at injection & $1.2 \times 10^{14}$ & \\
Maximum protons per cycle &
\end{tabular}

injection into PS2, which is compatible with the foreseen LHC luminosity upgrade schemes. For this purpose a lowpower $(<200 \mathrm{~kW})$ version of the SPL, the LP-SPL, will be installed to replace the PS Booster (PSB) providing a smaller-emittance higher-energy beam. While increasing the beam energy from 1.4 to $4 \mathrm{GeV}$, the SPL will also remove any reliability issues connected with the operation of the PSB. (ii) It will also provide an infrastructure that can be upgraded to cover the needs of all potential highpower proton users at CERN, such as EURISOL [9,10] or various neutrino production schemes (Superbeam [11] plus beta-beam [12], neutrino factory [13]). For high-power applications the high-power SPL (HP-SPL) will provide a $5 \mathrm{GeV}$, multi-megawatt beam, whose time structure can be tailored for each application. The potential for increased operational flexibility and beam power [14] of the SPL is the decisive argument in favor of a linac-based PS2 injector with respect to a solution based on a rapid cycling synchrotron (RCS) [15].

\section{B. LHC and PS2 requirements}

PS2 will be capable of providing twice the brightness of the "ultimate" LHC beam with $10 \%$ intensity reserve for beam losses in the following accelerator chain. This translates into $4.0 \times 10^{11}$ protons per LHC bunch at PS2 injection (assuming $25 \mathrm{~ns}$ bunch spacing and 10\% loss in PS2 itself) instead of presently $1.7 \times 10^{11}$. With 168 filled buckets (out of 180 ) PS2 requires $6.7 \times 10^{13}$ protons per cycle at an injection energy of $4 \mathrm{GeV}$. Table I summarizes the PS2 requirements for $\mathrm{LHC}$ operation.

The requirements for PS2 can be covered with a $1.2 \mathrm{~ms}$ pulse from the LP-SPL, carrying a $20 \mathrm{~mA}$ current.

\section{Low-power protons for radioactive ion beams (RIB)}

The LP-SPL is designed for a repetition rate of $2 \mathrm{~Hz}$ and the LHC will only use $20 \%$ of the available pulses. The additional beam out of the LP-SPL can be used for increased beam power to fixed target physics (with SPS) as mentioned in Table I, or for an increased proton flux to ISOLDE [16] or a first stage implementation of a
EURISOL type facility, respectively. The beam energy for ISOLDE/EURISOL is approximately $1.4 / 2.5 \mathrm{GeV}$ and is given by the layout of the SPL and the planned area for a possible installation of EURISOL on the CERN site (see Fig. 1).

A potential set of beam parameters, supplied by the LPSPL for ISOLDE/EURISOL, is given in Table II. Further beam power will be available for SPS fixed target physics or for potential neutrino applications (e.g. CNGS successor).

\section{High-power protons from the HP-SPL}

With an upgrade of the electrical and cryogenic infrastructure and by replacing the $2 \mathrm{~Hz}$ klystron modulators with $50 \mathrm{~Hz}$ modulators, the LP-SPL can be upgraded to a multi-MW proton injector. Since the upgrade also involves a higher beam current (40 instead of $20 \mathrm{~mA}$ ), the matching of the rf power couplers to the cavities can only be optimum for one current value. When matched to a $40 \mathrm{~mA}$

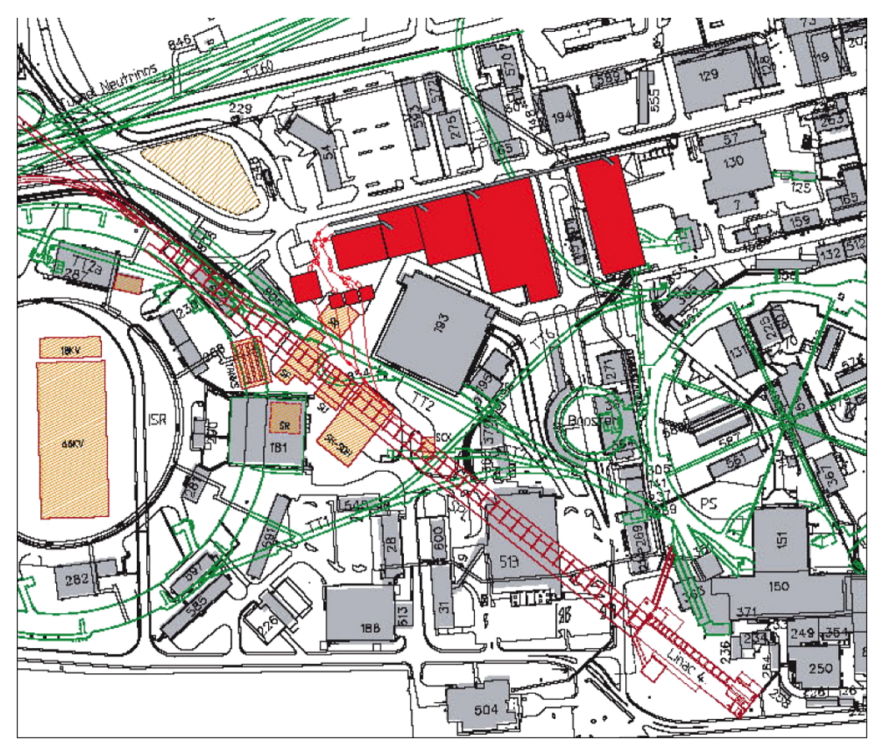

FIG. 1. (Color) Layout of a possible EURISOL facility at CERN (in red above building 193). 
TABLE II. LP-SPL beam parameters for LHC, PS2 fixed target, and radioactive ion beams.

\begin{tabular}{|c|c|c|c|c|}
\hline User & $\begin{array}{c}\text { PS2 } \\
(\mathrm{LHC})\end{array}$ & $\begin{array}{c}\text { PS2 } \\
\text { (fixed target) }\end{array}$ & ISOLDE II & $\begin{array}{l}\text { EURISOL } \\
\text { (first stage) }\end{array}$ \\
\hline Energy & $4 \mathrm{GeV}$ & $4 \mathrm{GeV}$ & $1.4 \mathrm{GeV}$ & $2.5 \mathrm{GeV}$ \\
\hline Average beam power & $20 \mathrm{~kW}$ & $40 \mathrm{~kW}$ & $42 \mathrm{~kW}$ & $75 \mathrm{~kW}$ \\
\hline Maximum repetition rate & $0.42 \mathrm{~Hz}$ & $0.42 \mathrm{~Hz}$ & $1.25 \mathrm{~Hz}$ & $1.25 \mathrm{~Hz}$ \\
\hline Pulse length & $0.6 \mathrm{~ms}$ & $0.96 \mathrm{~ms}$ & $1.2 \mathrm{~ms}$ & $1.2 \mathrm{~ms}$ \\
\hline Average pulse current & $20 \mathrm{~mA}$ & $20 \mathrm{~mA}$ & $20 \mathrm{~mA}$ & $20 \mathrm{~mA}$ \\
\hline ppp & $7.5 \times 10^{13}$ & $1.2 \times 10^{14}$ & $1.5 \times 10^{14}$ & $1.5 \times 10^{14}$ \\
\hline
\end{tabular}

current, the reflected power during $20 \mathrm{~mA}$ operation amounts to $11 \%$, a value that seems acceptable for the operation of the LP-SPL, especially since it avoids a replacement of the power couplers, when upgrading to the HP-SPL.

At present it appears that the two main potential users of such a beam are (i) EURISOL and (ii) a neutrino facility. In a conceptual design EURISOL prefers a low-energy (1$3 \mathrm{GeV}$ ) $\mathrm{cw}$ beam in order to minimize the beam-induced stress in the targets. Pulsed beams will generally result in shorter target lifetimes, but the effect can be mitigated if the repetition rate is high enough $(50 \mathrm{~Hz}$ or higher) and if the beam pulses are at least in the ms range [17]. For EURISOL the SPL can provide a multi-MW beam at $2.5 \mathrm{GeV}$. For the production of neutrinos several schemes are under study which can use the $5 \mathrm{GeV}$ SPL as the core part of the multi-MW proton driver [18]. Recently published results of the HARP experiment [19] have confirmed that an energy of $5 \mathrm{GeV}$ is well suited for the proton driver. In the case of neutrinos short pulses are preferred in order to minimize the number of injection turns in a subsequent accumulator. A detailed scheme based on the SPL has recently been designed [18]. A summary of options for high-power operation of the SPL is given in Table III.

\section{POSSIBLE LINAC ARCHITECTURES AND BEAM DYNAMICS}

\section{A. Introduction}

The frequency of the SPL has to be a multiple of $352.2 \mathrm{MHz}$, which is used in Linac4 in order to make use of a large stock of existing rf hardware (klystrons, waveguides, circulators, ...) at this frequency, which became free after the decommissioning of LEP. In order to limit the number of architectures to be compared, we have focused on the most promising solutions, which are: (i) the original cavity/cryomodule structure based on two families of 704.4 MHz five-cell cavities, which are described in the 2006 SPL design report [3]; (ii) a version using $4 \times$ the frequency of Linac4, 1408.8 MHz from $160 \mathrm{MeV}$ onwards; (iii) a mixture of spoke and elliptical cavities, using $352.2 \mathrm{MHz}$ spokes with normal conducting focusing elements, and then switching to $1408.8 \mathrm{MHz}$ elliptical cavities for the high-energy section of the linac.

Using $3 \times$ the base frequency of $352.2 \mathrm{MHz}$ was not considered, since $1056.6 \mathrm{MHz}$ is not used in any similar project and thus there is no technological advantage expected for this frequency. Also the option of mixing 704.4 and $1408.8 \mathrm{MHz}$ cavities was discarded because of the added $R \& D$ effort and the need for an additional rf system (klystrons, rf power distribution, etc.). In the following the assumptions for each architecture will be outlined and evaluated together with their respective beam dynamics performance.

\section{B. Fundamental assumptions}

In order to calculate the energy gain correctly, cavity fields including end-cell effects are fitted with simple functions describing the on-axis field of elliptical and spoke cavities. The maximum accelerating field is assumed to be $25 \mathrm{MV} / \mathrm{m}$ for elliptical cavities with a geometrical $\beta=1$ [20]. For $\beta<1$ the fields are scaled according to their ratio of peak field over accelerating field. The maximum power per cavity is limited in each option to $1 \mathrm{MW}$, a value that still seems realistic for the power coupler at all frequencies for the envisaged duty cycles of the SPL (see

TABLE III. Possible SPL beam parameters for high-power operation.

\begin{tabular}{lccc}
\hline \hline User & EURISOL & Neutrinos & EURISOL + neutrinos \\
\hline Energy & $2.5 \mathrm{GeV}$ & $5 \mathrm{GeV}$ & $2.5+5 \mathrm{GeV}$ \\
Average beam power & $3(6) \mathrm{MW}$ & $4 \mathrm{MW}$ & $4+4 \mathrm{MW}$ \\
Maximum repetition rate & $50 \mathrm{~Hz}$ & $50 \mathrm{~Hz}$ & $50 \mathrm{~Hz}$ \\
Pulse length & $1.2 \mathrm{~ms}$ & $0.4 \mathrm{~ms}$ & $0.8+0.4 \mathrm{~ms}$ \\
Average pulse current & $20(40) \mathrm{mA}$ & $40 \mathrm{~mA}$ & $40 \mathrm{~mA}$ \\
$p p p$ & $1.5(3) \times 10^{14}$ & $1.0 \times 10^{14}$ & $2.0 \times 10^{14}+1.0 \times 10^{14}$ \\
\hline \hline
\end{tabular}


TABLE IV. Lattice data of three possible SPL architectures.

\begin{tabular}{|c|c|c|c|}
\hline Architecture & $\begin{array}{c}\text { Nominal, } \\
\text { 704.4 MHz elliptical }\end{array}$ & $\begin{array}{c}1408.8 \mathrm{MHz} \\
\text { elliptical }\end{array}$ & $\begin{array}{c}352.2 \mathrm{MHz} \text { spoke }+1408.8 \mathrm{MHz} \\
\text { elliptical }\end{array}$ \\
\hline Geometrical $\beta$ & $0.65 / 0.92$ & $0.6 / 0.76 / 0.94$ & $0.67 / 0.8 / 0.94$ \\
\hline Cells per cavity & $5 / 5$ & $7 / 9 / 9$ & $4 / 5 / 9$ \\
\hline Cavity length $[\mathrm{m}]$ & $0.69 / 0.98$ & $0.45 / 0.73 / 0.90$ & $1.1 / 1.7 / 0.90$ \\
\hline Cavities per module & $6 / 8$ & $4 / 4 / 8$ & $3 / 4 / 8$ \\
\hline Cavities per period & $3 / 8$ & $2 / 4 / 8$ & $3 / 4 / 8$ \\
\hline Cavities per family & $42 / 200$ & $30 / 40 / 208$ & $27 / 24 / 216$ \\
\hline Focusing & FDO & FDO & FDO \\
\hline Doublet length [m] & 1.35 & 1.35 & $0.54 / 1.35$ \\
\hline Period length $[\mathrm{m}]$ & $6.1 / 14.4$ & $4.4 / 7.5 / 13.8$ & $5.4 / 9.0 / 13.8$ \\
\hline Accelerating gradient $[\mathrm{MV} / \mathrm{m}]$ & $19.4 / 24.2$ & $18.1 / 21.7 / 24.2$ & $8.5 / 9.5 / 24.2$ \\
\hline Transition energies $[\mathrm{MeV}]$ & $160 / 581$ & $160 / 357 / 884$ & $160 / 392 / 758$ \\
\hline Output energies [MeV] & 5122 & 5144 & 5075 \\
\hline Cavities in total & 242 & 278 & 267 \\
\hline Length [m] & 439 & 499 & 485 \\
\hline
\end{tabular}

Table III). It is assumed that the spacing between cavities and cryomodules is independent of the frequency and the number of cells. However, it was assumed that spoke cavities are used together with very compact normal conducting quadrupoles between cryomodules, while the elliptical cavity sections assume superconducting quadrupoles, which are part of the cryomodules. A detailed study about the pros and cons of having the quadrupoles inside of the cryomodules still has to be done. The basic lattice elements of all three architectures are given in Table IV.

\section{The nominal option: Five-cell elliptical cavities at 704.4 MHz}

This option is a slightly improved version of the layout, presented in the 2006 SPL report [3], which used two families of superconducting cavities $(\beta=0.65$ and $\beta=$ 1.0). Because of the fact that (single and multicell) cavities

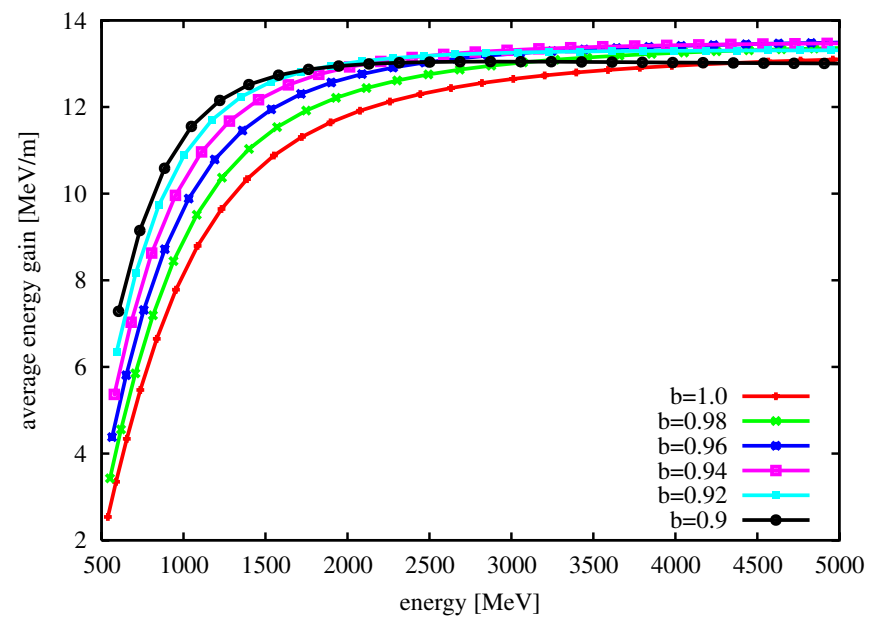

FIG. 2. (Color) Real-estate gradients for different geometric betas in the high-energy section of the SPL (corrected for lower fields at lower betas). achieve their maximum transit time factors always at velocities, which are slightly higher than their design beta, the high-energy section $(\beta=1)$ of the SPL is now exchanged with cavities using $\beta=0.92$ as their geometric beta. In Fig. 2 one can see the average energy gain in the high-beta section for different values of the geometric cavity beta. The values assume a cavity gradient of $25 \mathrm{MV} / \mathrm{m}$ at $\beta=1$ and are then scaled according to their reduced geometric beta.

The basic cavity layout of the high-energy section $(\beta=$ 0.92 ) comprises eight cavities and a quadrupoles doublet in one cryomodule, as shown in Fig. 3. In the low-energy section $(\beta=0.65)$ six cavities and two quadrupole doublets are housed in each cryomodule.

\section{The 1408.8 MHz option}

This option uses three families $(0.6,0.76$, and 0.94$)$ of superconducting cavities to accelerate the beam. The interest in this option comes from the fact that $1408.8 \mathrm{MHz}$ is close to the XFEL/ILC frequency, and the hope is that one could profit from existing cavity/cryomodule construction

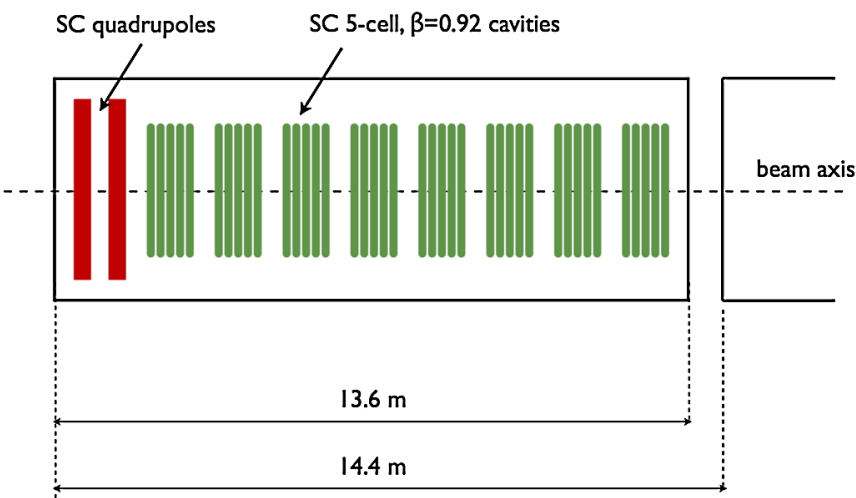

FIG. 3. (Color) SPL cavity/cryomodule layout in the highenergy section (five-cell, 704.4 MHz). 
infrastructure. Again a gradient of $25 \mathrm{MV} / \mathrm{m}$ is assumed at $\beta=1$ and then scaled for the different beta values. In order to be competitive to the $704.4 \mathrm{MHz}$ nominal solution, the number of cells has be increased, so that the filling factor (active cavity length over period length) remains approximately constant. For this reason and also to remain similar to the XFEL approach, a maximum number of nine cells per cavity was chosen. Since a higher number of cells also increases the phase slippage, three families of cavities become necessary for an efficient acceleration. For the same reason the low-beta section (0.6) has only seven cells per cavity.

The $4 \times$ frequency jump from Linac 4 to the SPL requires a careful matching of the beam, which is why the gradient and period length had to be reduced in the low-beta section. As a result of this and the slightly shorter cavity lengths (compared to the $704.4 \mathrm{MHz}$ option), the total length of the $1408.8 \mathrm{MHz}$ architecture is almost $15 \%$ longer than the nominal 704.4 MHz layout (see Table IV).

\section{E. The "mixed" option: spoke (352.2 MHz) + elliptical(1408.8 MHz)}

This layout uses the same $1408.8 \mathrm{MHz}$ nine-cell cavities as the previous one, but the two lower beta families are replaced by two families $(\beta=0.67$ and $\beta=0.8)$ of $352.2 \mathrm{MHz}$ spoke cavities. Using only one family of spoke cavities and using, for instance, two families of elliptical cavities at $1408.8 \mathrm{MHz}$ seems less interesting, because then a considerable amount of $R \& D$ would be spent on a very small number of spoke cavities. From the beam dynamics point of view this mixed layout seems interesting because the $4 \times$ frequency jump is shifted to higher energies, where the beam is much "stiffer" in the longitudinal plane and less sensitive to longitudinal disturbances. Gradients for the spoke cavities are assumed to be $8.5 \mathrm{MV} / \mathrm{m}(\beta=$ $0.67)$ and $9.5 \mathrm{MV} / \mathrm{m}(\beta=0.8)$, which are considered to be challenging but feasible. After beam matching across all sections, the layout was fixed to the one summarized in Table IV.

\section{F. Beam dynamics comparison}

Two iterations of beam dynamics simulations were done to compare the three different designs: (i) beam matching from Linac4 and across all transitions between all sections in the three layouts, then evaluation of the "nominal" beam envelopes for a matched beam, (ii) statistical runs including a $10 \%$ longitudinal mismatch plus a certain energy/phase jitter coming from Linac4 plus amplitude and phase errors coming from the klystrons in the SPL itself. The beam dynamics simulations were made with TRACE_WIN [21] and PATH [22] using 50000 particles for each run.

Figure 4 shows the evolution of the beam envelopes in all three planes for the nominal case and Table V lists the
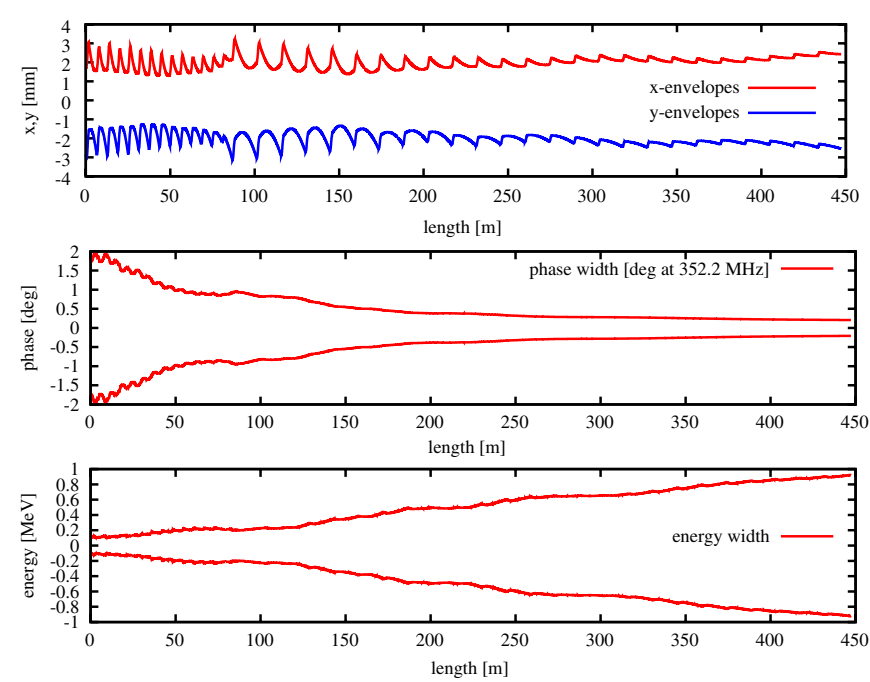

FIG. 4. (Color) Nominal beam envelopes in the nominal 704.4 MHz linac.

rms emittance evolution for all three layouts assuming a matched beam and no errors in the machine.

From the matched beam performance, one can see that the $1408.8 \mathrm{MHz}$ version shows the largest longitudinal rms emittance growth, while the spoke options seem to have the lowest overall rms emittance increase. The observed longitudinal sensitivity of the $1408.8 \mathrm{MHz}$ version is evident in the results of the error simulations shown in Table VI where a significant fraction of the runs are lossy. This data assumes the expected energy and phase jitter from Linac4 (including a 10\% longitudinal mismatch) plus a random error of $\pm 1 \% / \pm 1^{\circ}$ (maximum values of normal error distribution) on the klystron amplitude and phase in the SPL.

Table VI shows that the additional rms emittance growth due to statistical errors in the longitudinal plane is negligible. Also the energy and phase jitter values are comparable for all options. We attribute this behavior to a good longitudinal matching between the sections and we assume that if the matching sections for the frequency jumps were even longer, then one can also reduce the large longitudinal rms emittance growth that is observed for the nominal beam in the $1408.8 \mathrm{MHz}$ version [23].

\section{G. Summary on linac architectures and beam dynamics}

(i) For all elliptical cavities a maximum gradient of $25 \mathrm{MV} / \mathrm{m}$ (at $\beta=1$ ) was assumed and then scaled for the respective geometric betas. (ii) Compared with the 2006 SPL report [3] the $\beta=1$ cavities were replaced with cavities of slightly lower geometric beta values. (iii) It was found that the $4 \times$ frequency jumps, which are necessary to pass to $1408.8 \mathrm{MHz}$, show more sensitivity to longitudinal errors and require more space for matching from Linac4 to SPL. This is especially true if the transition takes place at low energy. (iv) The $1408.8 \mathrm{MHz}$ solution is 
TABLE V. Emittance growth for a matched beam in the three SPL layouts.

\begin{tabular}{lccc}
\hline \hline Architecture & Nominal, $704.4 \mathrm{MHz}$ elliptical & $1408.8 \mathrm{MHz}$ elliptical & $352.2 \mathrm{MHz}$ spoke $+1408.8 \mathrm{MHz}$ elliptical \\
\hline$\Delta \varepsilon_{x}[\%]$ & 5.6 & 6.3 & 1.5 \\
$\Delta \varepsilon_{y}[\%]$ & 8.2 & 7.8 & 5.3 \\
$\Delta \varepsilon_{z}[\%]$ & 6.8 & 12.1 & 2.5 \\
Losses & $\cdots$ & $\cdots$ & $\cdots$ \\
\hline \hline
\end{tabular}

TABLE VI. Additional rms emittance growth for simulations with statistical longitudinal errors, assuming $\pm 1 \% / \pm 1^{\circ}$ (uniform distribution) klystron errors in Linac4. Emittance errors are quoted as average $\pm 2 \times$ standard deviation.

\begin{tabular}{|c|c|c|c|c|c|c|}
\hline Architecture & \multicolumn{2}{|c|}{ Nominal, 704.4 MHz elliptical } & $1408.8 \mathrm{MH}$ & liptical & \multicolumn{2}{|c|}{ 352.2 MHz spoke $+1408.8 \mathrm{MHz}$ elliptical } \\
\hline \multicolumn{7}{|c|}{ Assuming $\pm 0.5 \% / \pm 0.5^{\circ}$ klystron errors in SPL (uniform distribution) } \\
\hline & Average & $\sigma$ & Average & $\sigma$ & Average & $\sigma$ \\
\hline$\Delta \varepsilon_{x}[\%]$ & 0.07 & 0.27 & 0.24 & 0.62 & 0.05 & 0.22 \\
\hline$\Delta \varepsilon_{y}[\%]$ & 0.18 & 0.26 & 0.10 & 0.38 & 0.09 & 0.24 \\
\hline$\Delta \varepsilon_{z}[\%]$ & 0.40 & 0.58 & 0.27 & 0.70 & 0.19 & 0.36 \\
\hline$\Delta E$ standard deviation $[\mathrm{MeV}]$ & $\cdots$ & 1.96 & $\cdots$ & 1.81 & $\cdots$ & 1.78 \\
\hline$\Delta \phi$ standard deviation $[\mathrm{deg}]$ & $\cdots$ & 0.26 & $\cdots$ & 0.30 & $\cdots$ & 0.30 \\
\hline Lossy runs [\%] & 0 & $\cdots$ & 9 & $\cdots$ & 0 & \\
\hline \multicolumn{7}{|c|}{ Assuming $\pm 1 \% / \pm 1^{\circ}$ klystron errors in SPL (uniform distribution) } \\
\hline & Average & $\sigma$ & Average & $\sigma$ & Average & $\sigma$ \\
\hline$\Delta \varepsilon_{x}[\%]$ & 0.21 & 0.41 & 1.02 & 1.11 & 0.24 & 0.49 \\
\hline$\Delta \varepsilon_{y}[\%]$ & 0.59 & 0.53 & 0.42 & 0.75 & 0.33 & 0.50 \\
\hline$\Delta \varepsilon_{z}[\%]$ & 1.13 & 1.32 & 1.90 & 1.88 & 0.81 & 0.76 \\
\hline$\Delta E$ standard deviation $[\mathrm{MeV}]$ & $\cdots$ & 3.78 & $\cdots$ & 3.48 & $\cdots$ & 3.45 \\
\hline$\Delta \phi$ standard deviation $[\mathrm{deg}]$ & $\cdots$ & 0.57 & $\cdots$ & 0.61 & $\cdots$ & 0.61 \\
\hline Lossy runs [\%] & 0 & $\cdots$ & 21 & $\cdots$ & 0 & $\cdots$ \\
\hline
\end{tabular}

almost $15 \%$ longer than the nominal $704.4 \mathrm{MHz}$ layout and shows the highest rms emittance growth in case of longitudinal errors. (v) For the spoke option a very compact lattice structure was assumed with normal conducting magnets between the SC cryomodules. Compared to the $1408.8 \mathrm{MHz}$ option the frequency jump is shifted to higher energies, which makes the frequency transition easier but which still results in a higher sensitivity to errors when compared to the nominal 704.4 MHz option. Using spoke cavities requires one to master two completely different types of superconducting cavities and only seems interesting if one cavity type is designed and constructed by a strong collaborator. (vi) The nominal solution, which follows the approach of the 2006 SPL report [3] results in the shortest linac and shows the best beam dynamics performance.

\section{PERFORMANCE OF SUPERCONDUCTING CAVITIES}

\section{A. Introduction}

This section contains the findings of [24]. The first key quantity defining the performance of superconducting cavities is the surface resistance, which describes the genera- tion of heat at the superconducting surface by the action of the rf magnetic field. The so-called BCS surface resistance is well understood and described quantitatively by the Mattis-Bardeen theory [25], applicable for the superconducting phase below a critical temperature $T_{c}$ and critical magnetic field $B_{c}$, characteristic features of the superconducting material [26]. The surface resistance depends on the temperature, frequency, and several material parameters.

The second key quantity relevant for the performance of superconducting cavities is the removal through the cavity wall into the refrigerant of the heat dissipated at the inner surface of a cavity. It depends largely on the thermal impedances of cavity wall and its interface to the liquid helium as coolant liquid. The heat transport is dependent on the thermal conductivity of the wall material and the interface heat conductance depending on the operational conditions (superfluid helium, normal boiling helium, power flow, etc.).

We call all these quantities "deterministic" because they are important for the generation and removal of heat, such as the rf frequency, operating temperature, thermal conductivity of the niobium, thermal impedance at the niobium-helium interface, etc. Their impact on perform- 
ance is well understood and they describe the "ideal" performance of the cavity.

However, the "real" performance of superconducting cavities is often degraded compared to the ideal case, due to the surface conditions both at the inner and outer surface of the cavity (surface asperities, normal conducting inclusions, field emission sites, electron-multipacting regions, trapped magnetic flux, chemical residues, surface roughness, etc.). Some of these parameters are difficult to control, because they are associated with potentially numerous microscopically small sites even one of which might be sufficient to degrade the performance of the cavity. Therefore we call them "stochastic."

The aim of the report is first to assess the deterministic parameters and second to compile the stochastic parameters and describe the impact of both on cavity performance. In a third part the electrical grid power requirements for the low- and high-power SPL are evaluated as depending on frequency and temperature.

\section{B. Electrical considerations}

Some preliminary remarks as to particularities of accelerating structures for protons shall be mentioned. As heavy particles, protons need a much longer accelerating length for attaining a velocity close to that of light. That is the reason why proton accelerators are composed of several stages of accelerating structures with a phase velocity synchronized with the actual velocity of the proton $v$ being smaller than light velocity $c$. These so-called low- $\beta$ structures $(\beta=v / c)$ entail, for a defined accelerating gradient $E_{a}$, larger surface magnetic fields $B_{p}$ and surface electric fields $E_{p}$ as compared to velocity of light $(\beta=1)$ accelerating structures. As the surface electric and magnetic fields determine the ultimate limit of the accelerating gradient, their dependence on $\beta$ must be taken into account for the assessment of the cavity performance.

The dependence of the surface fields on $\beta$ is a consequence to the Helmholtz condition for the transversal and longitudinal wave vector components $k_{r}$ and $k_{z}$, being valid close to the beam axis, $k_{r}^{2}+k_{z}^{2}=(\omega / z)^{2}$. An illustration is presented in Fig. 5, the numbers being taken from actual accelerating structures with variable $\beta$ [3]. A phenomenological fit of relevant cavity parameters is also indicated in Table VII, which is further used in the optimization calculations for the power requirements of the SPL.

\section{Deterministic parameters (ideal case)}

As a first step to assess the performance of superconducting cavities as required for the SPL under the most ideal conditions no intrinsic field dependence of the quality factor $Q$ is assumed. The heating of the inner wall of the cavity induces an apparent field dependence of $Q$, though, consequent to the temperature dependence of the BCSsurface resistance. The quality factor $Q$ will be simulated as a function of the accelerating gradient by taking into account only the deterministic parameters, which are the surface resistance $R_{s}$, the thermal conductivity [27] $\lambda$, the nucleate boiling heat transfer coefficient $\alpha$ for normal helium, and the Kapitza resistance $r_{K}$ for superfluid helium [24]. These quantities are dependent on the rf frequency, the liquid helium (lHe) bath temperature $T_{B}$, the temperature distribution as well as the power flow across the wall of
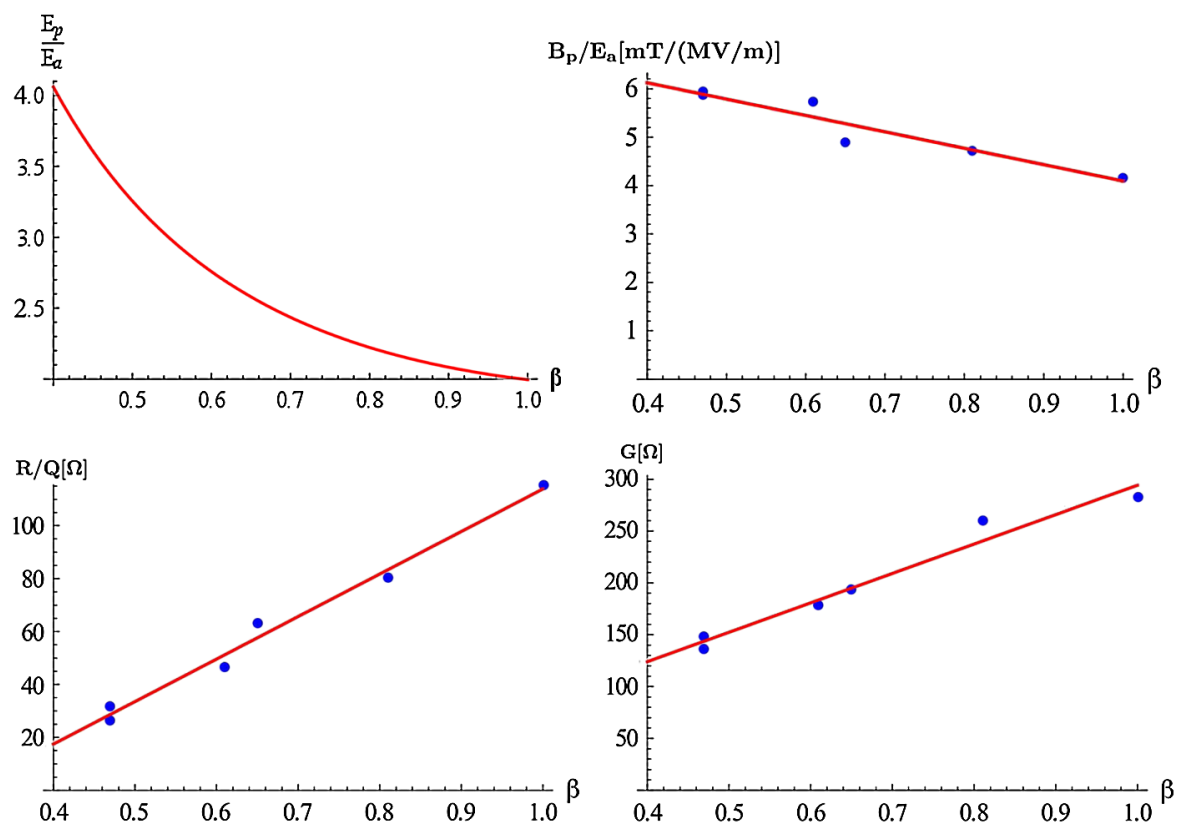

FIG. 5. (Color) Typical dependence of the surface electric field (upper left), the surface magnetic field (upper right), the $R / Q$ value (lower left), and the geometry factor (lower right) on the phase velocity $\beta$ of a low- $\beta$ elliptically shaped accelerating structure, as collected in Table VII. 
TABLE VII. Cavity parameters for different geometric $\beta$ values from various projects.

\begin{tabular}{lcccccc}
\hline \hline Project & $f[\mathrm{MHz}]$ & $\beta$ & $E_{p} / E_{a}$ & $B_{p} / E_{a}[\mathrm{mT} /(\mathrm{MV} / \mathrm{m})]$ & $R / Q^{\mathrm{a}}$ per cell $[\Omega]$ & $G[\Omega]$ \\
\hline RIA & 805 & 0.47 & 3.34 & 5.94 & 26.67 & 136.7 \\
TRASCO & 704.4 & 0.47 & 3.57 & 5.88 & 31.60 & 147.8 \\
SNS medium- $\beta$ & 805 & 0.61 & 2.71 & 5.72 & 46.50 & 179 \\
CEA/CNRS & 704.4 & 0.65 & 2.60 & 4.88 & 63.00 & 194 \\
SNS high- $\beta$ & 805 & 0.81 & 2.19 & 4.72 & 80.50 & 260 \\
TTF & 1300 & 1.0 & 2.0 & 4.16 & 115.11 & 283 \\
\hline
\end{tabular}

$E_{p} / E_{a} \approx 1.84 / \beta+1.17 \beta-1 B / E_{a}[\mathrm{mT} /(\mathrm{MV} / \mathrm{m})] \approx 7.5-3.38 \beta$

$R / Q[\Omega] \approx n_{\text {cells }}(160 \beta-47) G[\Omega] \approx 284 \beta+11$

${ }^{a} R / Q=\frac{V_{\text {acc }}^{2}}{\omega W}=\frac{V_{\text {ccc }}^{2}}{P_{d} Q_{0}}$ is used throughout this paper in the "linac definition" and is a surface-loss independent measure of the "effectiveness" of the cavity, i.e., "how much accelerating voltage one can get out of a certain amount of stored energy."

the cavity, and the temperature gradient $\Delta T$ at the liquid helium interface. The most relevant quantity is the BCS surface resistance $R_{\mathrm{BCS}}$, depending on the temperature $T$ at the inner wall of the cavity and the rf frequency $f$. It is approximated by the formula [28]

$$
R_{\mathrm{BCS}}(f, T)=A \cdot f^{2} \cdot e^{-\Delta /(k T)} / T .
$$

Several deterministic parameters other than mentioned are summarized in Table VIII. Their impact on cavity performance may be sufficiently reduced, as, for example, is the case for the trapped ambient magnetic field $B_{\text {ext }}$ during cooldown of the cavity. It contributes to the surface resistance as

$$
R_{s}^{m}(f, T)=B_{\mathrm{ext}} / B_{c}(T) \cdot R_{n}(f),
$$

$B_{c}$ being the critical magnetic field and $R_{n}$ the normal conducting surface resistance of niobium at low temperature. Also included in Table VIII are the cures required to keep the cavity performance at an acceptable level.

The simulation of the ideal performance of a cavity, as described by Eq. (1), is undertaken under $\mathrm{cw}$ conditions for the two different frequencies under consideration for the SPL during this study, 704.4 and $1408.8 \mathrm{MHz}$. A residual resistance $R_{\text {res }}$ of $21 \mathrm{n} \Omega$ is assumed for all cases resulting in a realistic quality factor $Q$ of $10^{10}$ at $704.4 \mathrm{MHz}$ and $2.2 \mathrm{~K}$.

In this simulation, the maximum gradient is limited by one of the following constraints, whatever gives the lowest value: either the surface magnetic field approaches the critical magnetic field of niobium,

$$
B_{p}(T)=200 \mathrm{mT} \cdot\left[1-\left(\frac{T}{T_{c}}\right)^{2}\right]
$$

or the heat flux density at the cavity wall-helium interface comes close to a critical value, $0.5 \mathrm{~W} / \mathrm{cm}^{2}$, by chance nearly identical for both superfluid and normal boiling helium. The variation of the temperature at the inner surface of the cavity with increasing power flow is taken into account and is responsible for the slow decrease of the quality factor $Q$ with the gradient (called " $Q$ slope" at intermediate $B_{p}$ or " $Q$ drop" at large $B_{p}$ ). It can be concluded that - for the ideal case - the accelerating gradients as envisaged for the SPL ( $25 \mathrm{MV} / \mathrm{m}$ for the $\beta=1$ cavity) can be obtained at all temperatures below $4.5 \mathrm{~K}$, however with a very small margin at $1408.8 \mathrm{MHz}$ and $4.5 \mathrm{~K}$ [24]. This statement is even more true because the SPL will be operated under pulse conditions resulting in an even lower average heat flux across the cavity wall [29].

\section{Stochastic parameters (real case)}

\section{Remarks on the obtainable accelerating gradient}

The real performance of a superconducting cavity, as observed experimentally, is not only dependent on the deterministic parameters, such as the helium bath tempera-

\begin{tabular}{|c|c|c|c|}
\hline Influencing parameter & Impact quantity & Physical explanation & Cure \\
\hline $\begin{array}{l}\text { External static } \\
\text { magnetic field } B_{\text {ext }}\end{array}$ & $\begin{array}{l}\text { Residual } \\
\text { resistance }\end{array}$ & $\begin{array}{l}\text { Creation of } \\
\text { vortices }\end{array}$ & $\begin{array}{l}\text { Shielding of ambient magnetic field } \\
\text { by Mu-metal }{ }^{\circledR} / \text { Cryoperm }{ }^{\circledR}\end{array}$ \\
\hline $\begin{array}{l}\text { Residual resistivity } \\
\text { ratio RRR }\end{array}$ & $\begin{array}{l}\text { BCS surface } \\
\text { resistance }\end{array}$ & $\begin{array}{c}\text { Mean-free } \\
\text { path dependence } \\
\text { of } R_{\mathrm{BCS}}\end{array}$ & $\begin{array}{c}\text { Annealing steps during ingot } \\
\text { production/after cavity } \\
\text { manufacture }\end{array}$ \\
\hline $\begin{array}{l}\text { Ratio peak magnetic } \\
\text { field to accelerating } \\
\text { gradient } B_{p} / E_{a}\end{array}$ & $\begin{array}{l}\text { Maximum } \\
\text { accelerating } \\
\text { gradient }\end{array}$ & $\begin{array}{l}\text { Critical magnetic } \\
\text { field as ultimate } \\
\text { gradient limitation }\end{array}$ & $\begin{array}{l}\text { Optimization of } \\
\text { cavity shape }\end{array}$ \\
\hline
\end{tabular}
ture or the rf frequency, but, to a large extent, on stochastic

TABLE VIII. Several additional deterministic parameters other than $R_{s}, f, T, \lambda, \alpha, r_{K}$. 
TABLE IX. Several stochastic parameters.

\begin{tabular}{|c|c|c|c|}
\hline Influencing parameter & Impact quantity & Physical explanation & Cure \\
\hline $\begin{array}{l}\text { Field emission sites } \\
\text { (foreign particles } \\
\text { sticking to the } \\
\text { surface, size, } \\
\text { density) }\end{array}$ & $\begin{array}{c}\text { Parasitic losses and } \\
\text { reduction of } Q / \text { accelerating gradient, } \\
\text { very large } X \text { radiation } \\
\text { HOM coupler quench }\end{array}$ & $\begin{array}{l}\text { Modified } \\
\text { Fowler-Nordheim theory }\end{array}$ & $\begin{array}{l}\text { Electropolishing; assembling in } \\
\text { dust-free air; rinsing with ultrapure } \\
\text { water (control of resistivity and } \\
\text { particulate content of outlet water) } \\
\text { and alcohol; high pressure ultrapure } \\
\text { water rinsing (ditto) } \\
\text { "He- processing"; } \\
\text { heat treatment at } 800-1400^{\circ} \mathrm{C}\end{array}$ \\
\hline $\begin{array}{l}\text { Secondary emission } \\
\text { coefficient } d\end{array}$ & Electron multipacting & $\begin{array}{l}\text { Theory of secondary } \\
\text { electron emission }\end{array}$ & $\begin{array}{l}\text { Rounded shape of cavity; } \\
\text { rinsing with ultrapure water; } \\
\text { bakeout rf processing }\end{array}$ \\
\hline Unknown & $Q(Q$-slope $/ Q$-drop/accelerating g & Unknown & Annealing $150^{\circ} \mathrm{C}$; Electropolishing \\
\hline $\begin{array}{l}\text { Metallic } \\
\text { normal-conducting } \\
\text { inclusions in } \mathrm{Nb}\end{array}$ & $\begin{array}{l}\text { Limitation of accelerating gradient } \\
\text { below theoretical value (quench) }\end{array}$ & $\begin{array}{l}\text { Local heating up until } \\
\text { critical temperature of } \mathrm{Nb}\end{array}$ & $\begin{array}{l}\text { Inspection of Nb sheets }(\text { eddy } \\
\text { current or SQUID scanning); } \\
\text { removal of defects }(\approx 1 \mathrm{~mm}) ; \\
\text { sufficiently large thermal conductivity } \\
(30-40[\mathrm{~W} /(\mathrm{mK})])\end{array}$ \\
\hline Residual resistance & $Q /$ accelerating gradient & To a large extent unknown & $\begin{array}{l}\text { Quality assurance control of a } \\
\text { multitude of parameters }\end{array}$ \\
\hline $\mathrm{Nb}-\mathrm{H}$ precipitate & $Q /$ accelerating gradient ( $Q$ disease) & $\begin{array}{l}\text { Lowering of } T_{c} / B_{c} \\
\text { at precipitates of } \mathrm{Nb}-\mathrm{H}\end{array}$ & $\begin{array}{l}T \text { control during chemical } \\
\text { polishing; degassing at } 700^{\circ} \mathrm{C} \text {; } \\
\text { fast cooldown }\end{array}$ \\
\hline
\end{tabular}

ones, such as the surface density of normal conducting inclusions leading to a thermal breakdown well below the theoretical value, microscopic particles that may emit electrons under the influence of large electric surface fields, chemical residues enabling multipactor discharges, etc.

The name "stochastic" expresses the fact that these parameters are difficult to control and that their impact on performance is not understood in all detail. As technical progress advances, and quality control measures take effect, the cause-impact relation will be understood, and cures will be found, consequent to which these parameters may become deterministic. Several stochastic parameters and associated cures are listed in Table IX.

These stochastic parameters do not allow a one-test prediction of the cavity performance, but are only valid for a large number of tests, which then will allow deducing the success yield. The smaller the width or steeper slope of the yield curves, the better is the mastering of the manufacture and processing steps. Data from the series production at Jefferson Lab of high- $\beta$ and low- $\beta$ superconducting cavities for the SNS spallation source are collected (top). The associated yield curves for the two types of cavities are shown at the bottom. The relatively small spread of results,

TABLE X. Summary of results in other labs.

\begin{tabular}{|c|c|c|c|c|}
\hline Laboratory & $\begin{array}{c}\left\langle E_{a}\right\rangle \\
{[\mathrm{MV} / \mathrm{m}]}\end{array}$ & $\begin{array}{c}\Delta E_{a} \\
{[\mathrm{MV} / \mathrm{m}]}\end{array}$ & $\begin{array}{c}\Delta E_{a} /\left\langle E_{a}\right\rangle \\
{[\%]}\end{array}$ & $\begin{array}{c}\left\langle E_{a}\right\rangle[\mathrm{MV} / \mathrm{m}] \text { at } 90 \\
(50)[\%] \text { processing yield }^{\mathrm{a}}\end{array}$ \\
\hline DESY $1.3 \mathrm{GHz}$ (all) & 28 & 5.2 & 19 & $22(28)$ \\
\hline ditto (quench) nine-cell & 30 & 6.9 & 23 & $23(30)$ \\
\hline ORNL/JLAB SNS $805 \mathrm{MHz}$ & & & & \\
\hline$\beta=0.616$-cell & 17.1 & 1.9 & 11 & $15(17)$ \\
\hline$\beta=1(\text { extrapolated })^{\mathrm{b}}$ & 23.0 & 2.6 & 11 & $20(23)$ \\
\hline$\beta=0.81$ 6-cell & 18.2 & 2.6 & 14 & $15(18)$ \\
\hline$\beta=1$ (extrapolated) & 20 & 2.8 & 14 & $16(20)$ \\
\hline
\end{tabular}

${ }^{\mathrm{a}} 90 \%$ or $50 \%$ yield is equivalent to $11 \%$ or $100 \%$ of reprocessing needed, respectively. The reprocessing rate in percent is equal to 100 (100-yield)/yield.

${ }^{b}$ The extrapolated value is calculated by means of the relation as in Fig. 5, depending on whether the field limitation is caused by a quench or a field emission. In the first case the surface magnetic field scaling relation is used, in the second case the surface electric field relation is applied. 
in particular, for the low- $\beta$ cavities, gives evidence on a well controlled production and processing chain. Results collected from the series production of superconducting cavities in other labs are summarized in Table X. As can be deduced from the last column of Table X, the yield depends very critically on the design accelerating gradient: The DESY $1.3 \mathrm{GHz}$ XFEL cavities allow for an accelerating gradient of $28-30 \mathrm{MV} / \mathrm{m}$, however at the expense of a production yield of 50\% for the presently applied processing sequence [30]. This means that on the average all cavities have to be reprocessed once. If a production yield of $90 \%$ is required, the average accelerating gradient for that yield would be smaller, about 22 to $23 \mathrm{MV} / \mathrm{m}$.

In conclusion, these test results show that, for the present state-of-the-art processing sequence, accelerating gradients of $16-23 \mathrm{MV} / \mathrm{m}$ ( $\beta=1$ cavities) for a production yield of $90 \%$ are possible. Higher accelerating gradients of 20-30 MV/m are also in reach, but at the expense of a lower production yield ( $\approx 50 \%$ ), which is equivalent to a higher reprocessing rate.

\section{Remarks on the obtainable quality factor $Q$}

Other stochastic parameters that mainly determine $Q$ are still unknown and under scientific debate, such as the residual resistance and the intrinsic field dependent part of the surface resistance. This must be distinguished from the slow decrease of the quality factor $Q$ with the gradient in the ideal case [24], which was due only to the exponential increase of the BCS surface resistance with the temperature of the inner wall of the cavity [see Eq. (1)], being heated up by the power flow across the wall. It turns out, however, that the experimentally observed $Q$ slopes are larger than simulated for the ideal case [31]; hence, the need to conjecture an intrinsic field dependent part of the surface resistance to account for the observed $Q$ slope. In [24] one finds typical experimental $Q\left(E_{a}\right)$ plots that exhibit these features: the low-field $Q$ is determined by the residual resistance and the $Q$ slope and $Q$ drop are clearly identified in the intermediate and high-field region, respectively.

As the stochastic parameters are not yet understood and, hence, cannot be modeled as in the ideal case, an alternative approach is chosen for the purpose of this study. We analyze a large variety of data for the surface resistance (452 measurements) obtained with cavities of different shape, temperature, frequency, and rf field amplitude. The shapes cover elliptical cavities, spoke resonators, and quarter wave resonators. The temperature ranges from 1.4 to $4.6 \mathrm{~K}$. The frequency spans from 80 to
$3000 \mathrm{MHz}$. The surface fields extend up well above $100 \mathrm{mT}$ at low temperatures; however, the measurements are typically limited to a few tens of $[\mathrm{mT}]$ at normal boiling helium temperature, because of power dissipation. Analyzing measured data has the advantage that the backreaction of the power flow across the cavity wall on the temperature at the inner wall is a priori taken into account. We obtain the parametrization of $Q$ by the phenomenological Ansatz for the surface resistance:

$$
\begin{aligned}
R_{s}\left(f, B_{p}, T\right)= & R_{\mathrm{BCS}}(f, T)+R_{\mathrm{res}}+R_{s}^{m}\left(f, B_{p}, T\right) \\
& +R_{s}^{\prime}\left(f, B_{p}, T\right) .
\end{aligned}
$$

The different components in Eq. (4) are the BCS surface resistance $R_{\mathrm{BCS}}$ [see Eq. (1)], the residual resistance $R_{\text {res }}$, the surface resistance $R_{s}^{m}$ that depends on the trapped magnetic flux [32], and the field dependent surface resistance $R_{s}^{\prime}$.

There is confirmed evidence that $R_{s}^{\prime}$ factorizes into a field dependent and a temperature dependent part $[33,34]$. The field dependent part may be described by a quadratic fit in $B_{p}$ and an exponential fit for the bath temperature $T$ :

$$
\begin{aligned}
R_{s}= & R_{\mathrm{res}}+a \cdot f^{n 1} \frac{\exp \left(-\Delta_{1} / T\right)}{T}+b \cdot f^{n 2} \frac{\exp \left(-\Delta_{2} / T\right)}{T} \\
& \cdot\left(1+\alpha_{2} \cdot B^{2}\right)
\end{aligned}
$$

with $R_{s}$ and $R_{\text {res }}$, as usual, measured in $\mathrm{n} \Omega, f$ in [GHz], $T$ in $[\mathrm{K}]$, and $B$ in [mT]. The maximum likelihood fits are presented in [24]. The resulting fit parameters are given in Table XI:

It is reassuring that the parameters describing the BCS surface resistance, such as $a, n_{1}$, and $\Delta_{1}$, turn out as expected.

The quality factor $Q$ is inversely proportional to the total surface resistance $R_{s}$ [see Eq. (4)], with $G$ the geometry factor, which has a numerical value around $280 \Omega$ for elliptical $\beta=1$ cavities.

\section{Simulation results for $Q\left(E_{a}\right)$ by the phenomenological method}

Subsequently, the phenomenological method, summarized by Eq. (5), was applied for two frequencies, in debate for the SPL, 704.4 and $1408.8 \mathrm{MHz}$, and for a variety of bath temperatures, ranging from that of superfluid helium to that of normal boiling helium. The results are shown in Fig. 6.

The following conclusions may be drawn. The design $Q$ of $10^{10}$ at an accelerating gradient of $E_{a}=25 \mathrm{MV} / \mathrm{m}$ $\left(B_{p}=100 \mathrm{mT}\right)$ for $\beta=1$ cavities is attainable provided

TABLE XI. Fit parameters for Eq. (5).

\begin{tabular}{lccccccc}
\hline \hline$R_{\text {res }}$ & $a$ & $n_{1}$ & $\Delta_{1}$ & $b$ & $n_{2}$ & $\Delta_{2}$ & $\alpha_{2}$ \\
\hline $2.0 \pm 0.3$ & $(78 \pm 8) \times 10^{3}$ & $2.15 \pm 0.15$ & $18.0 \pm 0.3$ & $2150 \pm 150$ & $1.02 \pm 0.06$ & $11.3 \pm 0.2$ & $(0.85 \pm 0.07) \times 10^{3}$ \\
\hline \hline
\end{tabular}



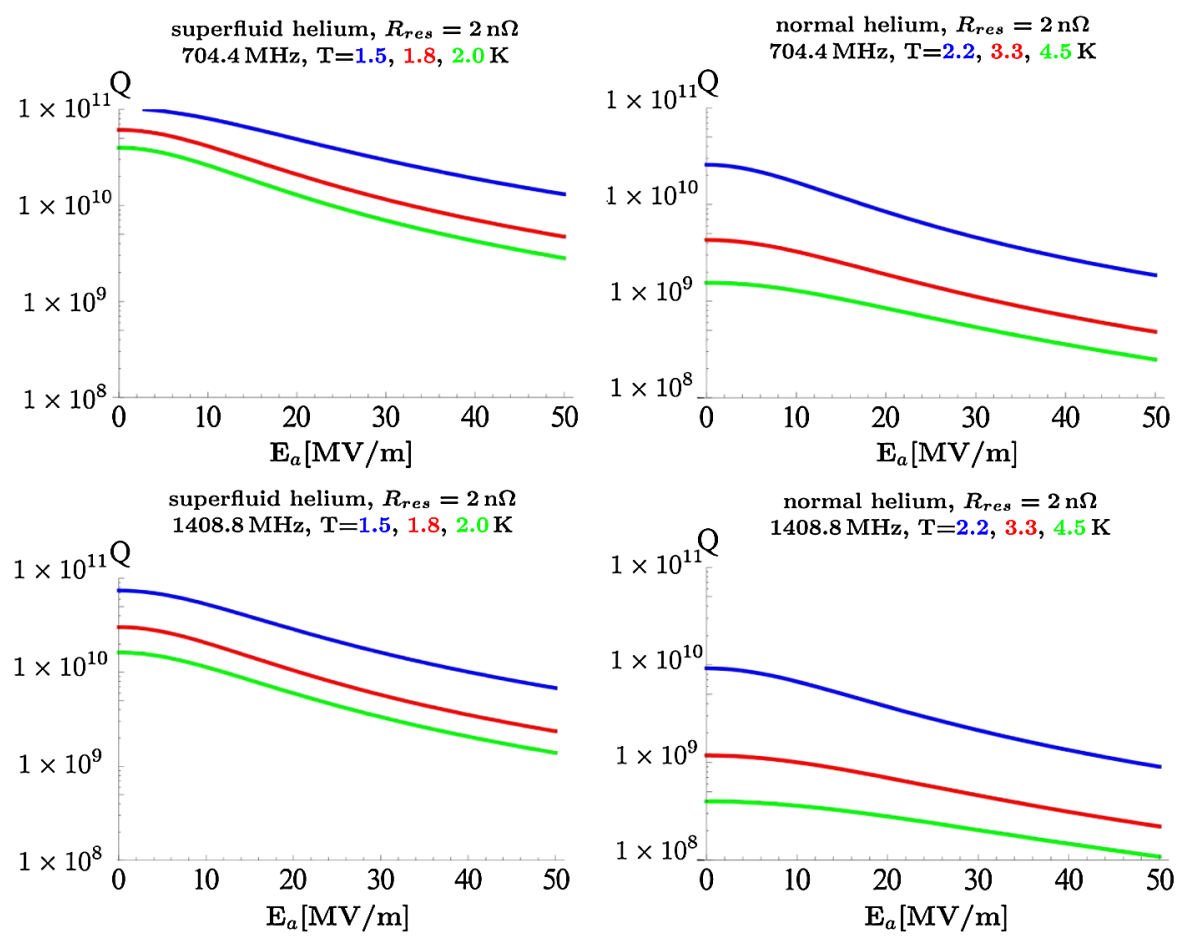

FIG. 6. (Color) Real performance of SPL-type $\beta=1$ superconducting cavities, as summarized by Eq. (5), at different frequencies, 704.4 MHz (top) and 1408.8 MHz (bottom), and several bath temperatures for cooling with superfluid helium (left) and normal boiling helium (right).

that the bath temperature is lowered to $1.9 \mathrm{~K}$ for 704.4 MHz, and to $1.6 \mathrm{~K}$ for $1408.8 \mathrm{MHz}$, respectively. Both operating bath temperatures require a cryogenic installation for superfluid helium.

For $\beta=0.65$ cavities, applying the formulas listed in Table VII, for the same surface magnetic fields (100 mT) and temperatures as above, the accelerating gradients and quality factors $Q$ are somewhat lower, $E_{a}=19 \mathrm{MV} / \mathrm{m}$, $Q=7 \times 10^{9}$.

Operation under normal boiling helium conditions, say $4.5 \mathrm{~K}$, is yet not excluded. The quality factor $Q$ at $E_{a}=$ $25 \mathrm{MV} / \mathrm{m}$ would be lower according to the simulation, $5 \times 10^{8}$ and $2 \times 10^{8}$ for 704.4 and $1408.8 \mathrm{MHz}$, respectively, however keeping in mind that reliable experimental data at these fields and temperatures are lacking for the time being. Operation in this regime would be at the expense of a considerable increase of the total electrical power consumption for both the low- and high-power SPL.

TABLE XII. Expected parameters of superconducting cavities at 704.4 and $1408.8 \mathrm{MHz}$.

\begin{tabular}{lcccc}
\hline \hline$f[\mathrm{MHz}]$ & $\beta_{\text {geom }}$ & $(R / Q)[\Omega]$ & $Q_{0}\left[10^{9}\right] 4.5 / 2 \mathrm{~K}$ & $E_{a}[\mathrm{MV} / \mathrm{m}]$ \\
\hline 704.4 & 0.65 & 285 & $0.30 / 5.8$ & 19.4 \\
704.4 & 0.92 & 501 & $0.40 / 7.7$ & 24.2 \\
1408.8 & 0.60 & 441 & $0.10 / 2.3$ & 18.1 \\
1408.8 & 0.76 & 671 & $0.12 / 2.5$ & 21.7 \\
1408.8 & 0.94 & 931 & $0.15 / 3.2$ & 24.2 \\
\hline \hline
\end{tabular}

This finding is detailed in the next section. The expected cavity properties for cavities at 704.4 and $1408.8 \mathrm{MHz}$ are listed in Table XII.

\section{E. Simulation of the total electrical power consumption}

The simulation was performed by applying Eq. (5), as graphically displayed in Fig. 6, by using the typical design parameters for the high- and low-power SPL. The results of the simulation for the high-power SPL are displayed in Fig. 7. The simulation was made for the SPL $\beta=1$ section assuming $25 \mathrm{MV} / \mathrm{m}, 40 \mathrm{~mA}$ pulse current, beam loss of $1 \mathrm{~W} / \mathrm{m}$, cavity filling time of $0.72 \mathrm{~ms}$, and a residual resistance of $R_{\text {res }}=3 \mathrm{n} \Omega$.

It should be understood that some numbers used in this simulation are average numbers and therefore somewhat arbitrary. The efficiency of the whole rf chain, from the electrical power grid to the cavity proper, is taken as $\eta_{\mathrm{rf}}=$ $40 \%$. Similarly, the cryogenic figure of merit (percent Carnot) was assumed as $\eta_{\mathrm{td}}=20 \%$. The total occupancy of "active" length (total length of cavities providing accelerating gradient) to real-estate length is taken as $\eta_{\text {real estate }}=50 \%$. Finally, the cryogenic static losses, defined as the total cryogenic power deposit at low temperature except for the rf dissipation, are approximated to $P_{\text {cst }}=1 \mathrm{~W} / \mathrm{m}$, independent of the operating temperature.

In Fig. 7, the total power consumption (thick lines) is split into the principal components, i.e., the electrical power taken from the grid needed to operate the rf equip- 

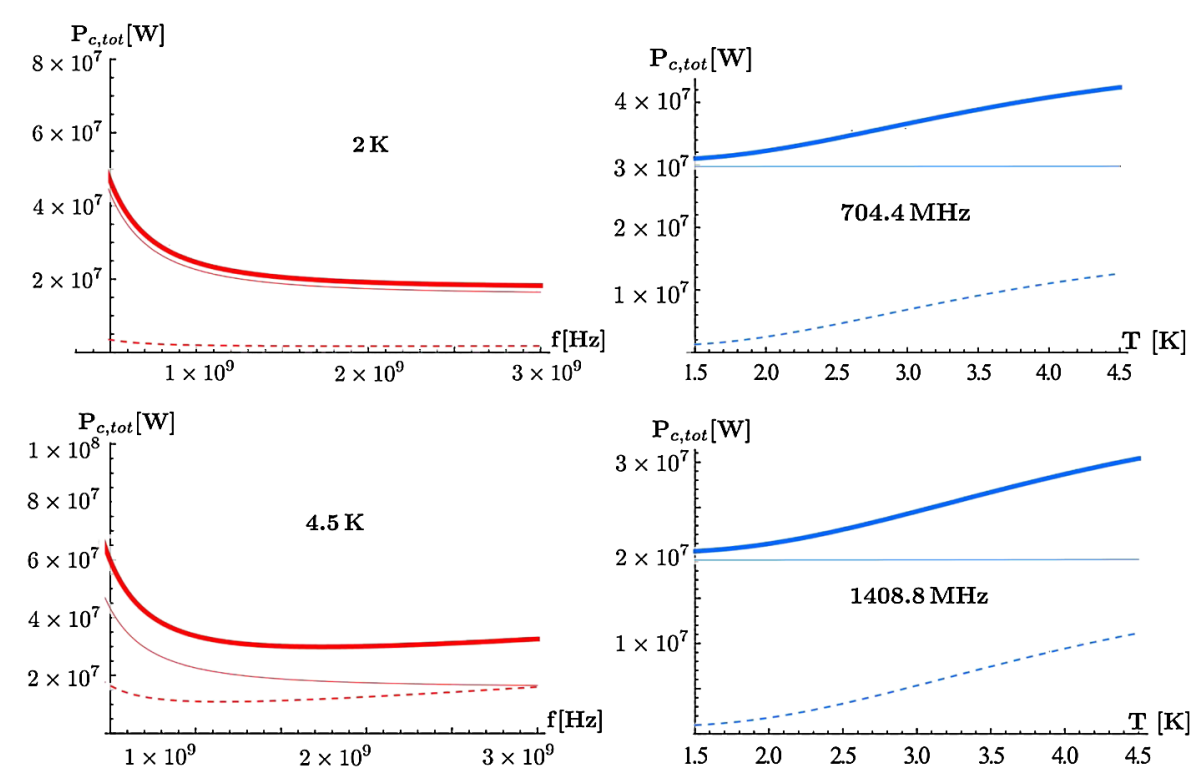

FIG. 7. (Color) Power requirements of the high-power SPL. The left column (red lines) depicts the power consumption as a function of frequency at two bath temperatures, 2.0 and $4.5 \mathrm{~K}$. The right column (blue lines) depicts the power consumption as a function of the bath temperature at two different frequencies, 0.7 and $1.4 \mathrm{GHz}$. The simulation is performed by applying Eq. (5) and using the parameters for the high- and low-power SPL as indicated in the above boxes at the right. Thick lines indicate the total electrical grid power, thin lines that for the rf system, and dashed lines that for the cryogenic system.

ment and to provide the beam power (thin continuous line), and the electrical power taken from the grid required for the cryogenic installation (thin dashed line).

It can be concluded that the minimum total electrical power consumption, both for the low- and high-power SPL, can be achieved for an operating temperature close to $2 \mathrm{~K}$ or somewhat below. This statement is valid for both frequencies under study, 704.4 and 1408.8 MHz.

It can also be concluded that the total electrical power consumption at $4.5 \mathrm{~K}$, should operation with normal boiling helium be desired, is by about $60 \%-80 \%$ larger than the optimum close to $2 \mathrm{~K}$.

As to the different frequencies under study, 704.4 and $1408.8 \mathrm{MHz}$, the total electrical power consumption is by about $40 \%$ or $50 \%$ larger at $704.4 \mathrm{MHz}$ than at $1408.8 \mathrm{MHz}$, for the low- and high-power SPL, respectively. The reason is the larger stored energy and longer filling and decay times, which for pulsed operation lead to larger dissipation both to the $\mathrm{lHe}$ and room temperature loads.

\section{F. Conclusions}

The study conducted so far has these main results: The SPL design parameters for a quality factor of $Q=10^{10}$ and the accelerating gradient (equivalent $25 \mathrm{MV} / \mathrm{m}$ for $\beta=1$ cavities) are realistic and can be achieved with present state-of-the-art manufacture and surface processing procedures, however either at the expense of some retreatment or by applying improved processing methods such as electropolishing.
To obtain these design parameters, a cryogenic system operating with superfluid helium is required. The total power consumption of the low- and high-power SPL, for the two frequencies under study ( 1408.8 and $704.4 \mathrm{MHz}$ ) is minimized for an operating temperature of $2 \mathrm{~K}$ and below.

Because of the difference in stored energy in the cavities, the total power consumption is smaller at $1408.8 \mathrm{MHz}$ operating frequency than at $704.4 \mathrm{MHz}$ (about one third for the HP-SPL). However, as outlined in the Introduction, there are other parameters also relevant for the choice of the operating frequency.

\section{SCALING OF FUNDAMENTAL CHARACTERISTICS OF SUPERCONDUCTING CAVITIES WITH FREQUENCY AND NUMBER OF CELLS}

\section{A. Introduction}

In this section we will consider first the straightforward linear geometric scaling of a cavity and its direct ancillaries such as main coupler (MC) and higher order mode (HOM) coupler(s). Assuming the same accelerating gradient, a doubling of the frequency from 704.4 to $1408.8 \mathrm{MHz}$ means that one has to double the number of cells per cavity in order to avoid a substantial lengthening of the linac. In the considered range of frequencies (704.4$1408.8 \mathrm{MHz}$ ) the gain in expensive high RRR niobium due to this scaling is more or less compensated by the additional necessary working steps (see e.g. [35]) due to the doubling of the cells. 
However, this cell doubling has non-negligible disadvantages on the technical side. One of these drawbacks comes from the principal field profile of modes with more resonators (cells), the second that manufacturing tolerances and end-cell corrections weigh heavier for longer structures. We will examine these two points in a second part.

The purpose of this section is to derive a qualitative comparison between the effects or "dangers" of HOMs to the beam when choosing 704.4 or $1408.8 \mathrm{MHz}$. A quantitative study that determines which damping the HOM couplers have to provide will follow. The problem will be approached in three steps: (i) doubling of frequency, (ii) doubling of cells, (iii) doubling of frequency and cells. Finally we will look at the influence of imperfections during the manufacturing process. For the whole section we assume that the bunches come with a repetition rate of $352.2 \mathrm{MHz}$, which is given by the normal conducting front end of the SPL.

\section{B. Linear cavity scaling (keeping the number of cells per cavity constant)}

If we consider the doubling of the frequency from 704.4 to $1408.8 \mathrm{MHz}$ and a linear geometrical scaling (see Fig. 8), then any length becomes $1 / 2$ as long, any area $1 / 4$ as large, and any volume $1 / 8$ as big. It follows easily that all cavity mode frequencies double, and for identical fields at equivalent cavity locations the longitudinal voltage gets $1 / 2$ as big and the stored energy becomes $1 / 8$. Injecting these relations in the definition of the longitudinal beam-cavity coupling constant $(R / Q)_{\|}$one sees that it remains invariant under this scaling

$$
\left(\frac{R}{Q}\right)_{\|}=\frac{V^{2}}{\omega U_{\mathrm{st}}} \quad \text { (linac definition) }
$$

invariant, excitation independent.

For the same local fields the current picked up by a pure antenna with $1 / 4$ surface for pickup but at twice the frequency becomes half, hence-with the line impedance being invariant - the coupled power becomes $1 / 4$. In analogy a pure loop coupler with $1 / 4$ coupling loop cross section but twice the frequency picks up $1 / 2$ the voltage, hence $1 / 4$ the power. Finally, any losses in resistive material are proportional to the area, hence they also scale to $1 / 4$. This means also that any imaginable hybrid coupler (as the real ones always are) picks up $1 / 4$ of the power. Therefore (having 1/4 of the external power, $1 / 8$ of the

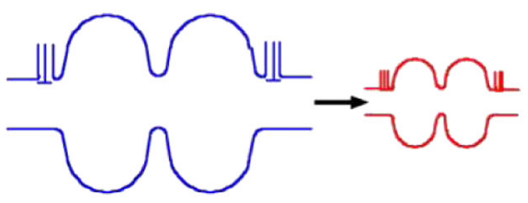

FIG. 8. (Color) Linear cavity scaling. stored energy, and twice the frequency) also the coupling constant $Q_{\text {ext }}$ of any coupling device remains invariant:

$$
Q_{\mathrm{ext}}=\frac{\omega U_{\mathrm{st}}}{P_{\mathrm{ext}}} \quad \text { invariant, excitation independent. }
$$

The voltage induced in a cavity (mode) by the passage of a particle of charge $q$ is given [follows from the definition of $(R / Q)_{\|}$by energy conservation] by

$$
\Delta V_{\text {ind }}=\frac{1}{2} q \omega\left(\frac{R}{Q}\right)_{\|}
$$

hence doubles. For twice the number of cells or when counting the longitudinal voltage induced per length (short range wake), $\boldsymbol{\Delta} \boldsymbol{V} / \boldsymbol{L}$ scales by a factor 4 . The longitudinal (long range) impedance per cavity is defined as $Z_{\|}($cav $)=$ $0.5 \cdot(R / Q)_{\|} \cdot Q_{\text {ext }}$ and remains invariant, however the impedance per length $\boldsymbol{Z}_{\|} / \boldsymbol{L}$ (or the impedance when doubling the number of cells) increases by a factor of 2 .

For a scaling of the dipole wakes, one has to keep the transverse particle offset from the beam axis constant. Longitudinal fields of dipole modes disappear on the axis and increase (in first order) linearly with off-axis parameter $x$ (see Fig. 9). Because of the double distance-relative to the cavity size - from the axis the longitudinal local fields become twice as big, while the integration path length gets halved, i.e., the measured acceleration voltage (for identical local fields at equivalent locations) is the same. The longitudinal $(R / Q)_{\|}(x)$ of a dipole mode scales hence by a factor of 4 (scaling of stored energy and frequency). Now exploiting the excitation invariance of $(R / Q)_{\|}$one can calculate the voltage induced by an off-axis beam current $I_{B, \text { rf }}$ as $V_{\|}(x)=0.5 \cdot I_{B, \text { rf }} \cdot(R / Q)_{\|} \cdot Q_{\text {ext }}$ scaling by a factor 4 or the voltage per length $V_{\|}(x) / L$ scaling by a factor 8 . Applying the Panofsky-Wenzel theorem and approximating $d V_{\|}(x) / d x$ by $V_{\|}(x) / x$, one gets

$$
\Delta p_{x}=-\frac{i \cdot e}{\omega} \cdot \frac{\delta V_{\|}}{\delta x} \approx-\frac{i \cdot e}{\omega} \cdot \frac{V_{\|}}{x} .
$$

This means that the transverse momentum kick per length $L$ increases by a factor 4 during the scaling or $\left(\boldsymbol{Z}_{\perp} / \boldsymbol{L}\right)$ increases by a factor 4 .

A linac does not have the quasiperiodicity condition with the revolution frequency as circular machines, hence an instability can be excited at any frequency, it must not be close to a machine line (here multiples of the bunch frequency of $352.2 \mathrm{MHz}$ ). Therefore the above statement

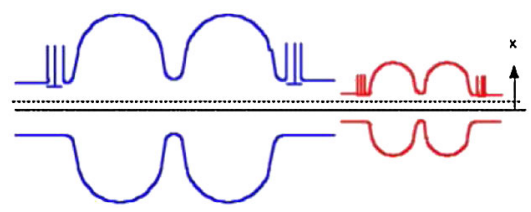

FIG. 9. (Color) Definition of off-axis parameter $x$. 
TABLE XIII. HOM parameter scaling for linear frequency scaling (same number of cells).

\begin{tabular}{lcc}
\hline \hline Parameter & 704.4 MHz, five-cell & $1408.8 \mathrm{MHz}$, five-cell \\
\hline$V_{\text {ind }}($ monopoles, short range, per cavity) & 1 & $\times 2$ \\
$Z_{\|} / L$ (monopoles, long range) & 1 & $\times 2$ \\
$\Delta p_{x}$ (dipoles, long range, per cavity) & 1 & $\times 2$ \\
$Z_{\perp} / L$ (dipoles, long range) & 1 & $\times 4$ \\
$I_{\mathrm{BBU}}$ & 1 & $/ 4$ \\
\hline \hline
\end{tabular}

means that the transverse beam breakup threshold current - the most critical threshold in linacs-is only $1 / 4$ at $1408.8 \mathrm{MHz}$ compared to $704.4 \mathrm{MHz}$. If this threshold is critical, a stronger mode damping by decreasing $Q_{\text {ext }}$ is the only way out.

Another aspect is the power deposition in (longitudinal) HOMs. The longitudinal impedance per length increases already by a factor 2 but for high- $Q$ resonances the frequency relation between resonance and essential machine lines becomes important. When scaling the cavity frequency, the beam spectrum remains invariant, hence the two cases are different and not immediately scalable problems. However, it can be said that the machine lines appear relatively twice as dense for a $1408.8 \mathrm{MHz}$ cavity compared to $704.4 \mathrm{MHz}$ cavity. This can be seen by anadmittedly malicious-example: a $\mathrm{HOM}$ in a $704.4 \mathrm{MHz}$ cavity at $1225 \mathrm{MHz}-3.5$ times $352.2 \mathrm{MHz}$-is exactly between two principal machine lines, hence presents no problem. This mode scales to $2450 \mathrm{MHz}-7$ times 352.2 $\mathrm{MHz}$ - and precisely hits a principal machine line in a $1408.8 \mathrm{MHz}$ cavity. This shows that all HOMs in a 704.4 MHz cavity sitting between machine lines scale on a machine line in a $1408.8 \mathrm{MHz}$ cavity. All HOMs on machine lines in a $704.4 \mathrm{MHz}$ cavity scale also onto a machine line in a $1408.8 \mathrm{MHz}$ cavity, hence the relative danger is twice as big. The only advantage of the higher frequency might be that the bunch-form factor might drop down significantly. However, using information from [36], it turns out that up to $5 \mathrm{GHz}$ the bunch form factor is above $80 \%$, giving no compensating advantage for the higher frequency.

Looking at a "typical strong" $\mathrm{HOM}$ (e.g. similar TM011) at $(R / Q)=100 \Omega, Q_{\text {ext }}=50000$ precisely on a machine line (worst case) with $40 \mathrm{~mA}$ "on-train" current. Then the on-train power is about $8 \mathrm{~kW}-0.9 \mathrm{kV}$ peak voltage on a $50 \Omega$ transmission line - but for a duty factor of $8 \%$ this is $650 \mathrm{~W}$, hence still no serious problem (provided that there are not many more such modes). For comparison, a similar mode in an LHC cavity with a $15 \times$ higher current and $100 \%$ duty cycle would couple 1.8 MW which could not be handled anymore. For SPL it should not be forgotten that this HOM power had to be supplied by the accelerating mode, lowering the overall efficiency, and presents a beam-loading problem concerning the acceleration stability along the batch.
One might try to "shift" HOM frequencies during the design phase but this is not easy when considering "all" modes; one improvement often results in a degradation elsewhere and the geometrical range of possible (elliptic) cavity modifications is very limited. Also the ILC/TESLA or FLASH case with "one rare big" bunch (where fields get some time to decay) does not scale directly to the SPL case with "many consecutive small" bunches.

In summary, one can establish the scaling parameters in Table XIII for the case of linear scaling, keeping the number of cells constant.

\section{Doubling of the cell number (perfect structures)}

In this section we add the effects of doubling the cell number to the effects of the previous section (frequency scaling) as illustrated in Fig. 10. This means that we keep the cell dimensions and (single-cell) frequency fixed, and vary only the number of cells in the cavity. In this spirit then also all HOM couplers are of the same make.

In every HOM field pattern existing in a single-cell cavity there exist $N$ incarnations in a cavity with $N$ such cells, indexed by $m=1$ to $m=N$ (or $m=0$ to $m=N-$ 1 according to the field pattern). For a cell-to-cell coupling $K$ and the single-cell angular frequency $\omega_{0}$ the frequency of $N$-cell cavity mode $m$ follows the relation (see e.g. [37])

$$
\omega_{m}^{2}=\omega_{0}^{2} \cdot\left\{1+2 K \cdot\left[1-\cos \left(\frac{\pi \cdot m}{N}\right)\right]\right\}
$$

having zero slope at both ends. Hence, the difference between adjacent mode frequencies approaches zero for increasing $N$. Figure 14 shows such mode frequencies (as dots on this line) in the case of $N=5$ and $N=10$ cells. Figure 11 illustrates the behavior of $\left(\omega_{m} / \omega_{0}\right)^{2}$ for a large coupling constant of $K=0.2$.

The (not normalized) cell-to-cell field-pattern amplitude [the cell field map corresponding (about) to the single-cell map apart in the coupling area between cells] in cell $n(1 \leq$ $n \leq N$ ) for mode $m$ is

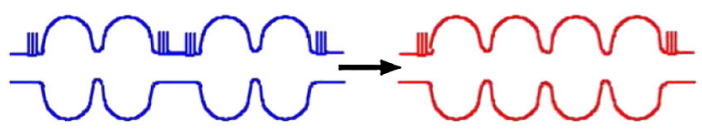

FIG. 10. (Color) Doubling of the cell number. 


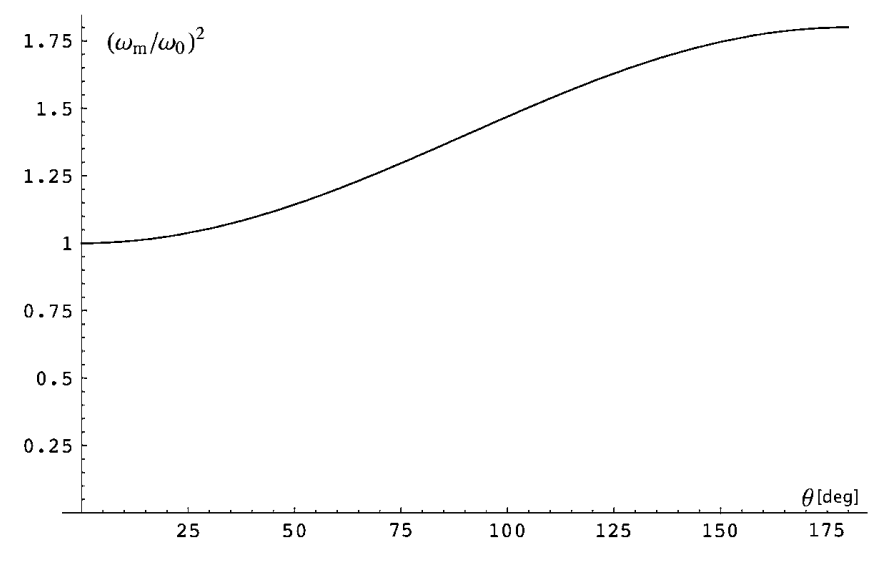

FIG. 11. $\left(\omega_{m} / \omega_{0}\right)^{2}$ as a function of $\Theta\left[^{\circ}\right]=180^{\circ} \cdot m / N$ for the (very large) coupling constant $K=0.2$.

$$
a_{n}^{(m)}=\sin \left(\frac{\pi \cdot m}{2 N} \cdot(2 N-1)\right)
$$

which can be combined as amplitude vector for mode $m$ as

$$
A^{(m)}=\left\{a_{1}^{(m)}, a_{2}^{(m)}, \ldots, a_{N}^{(m)}\right\}
$$
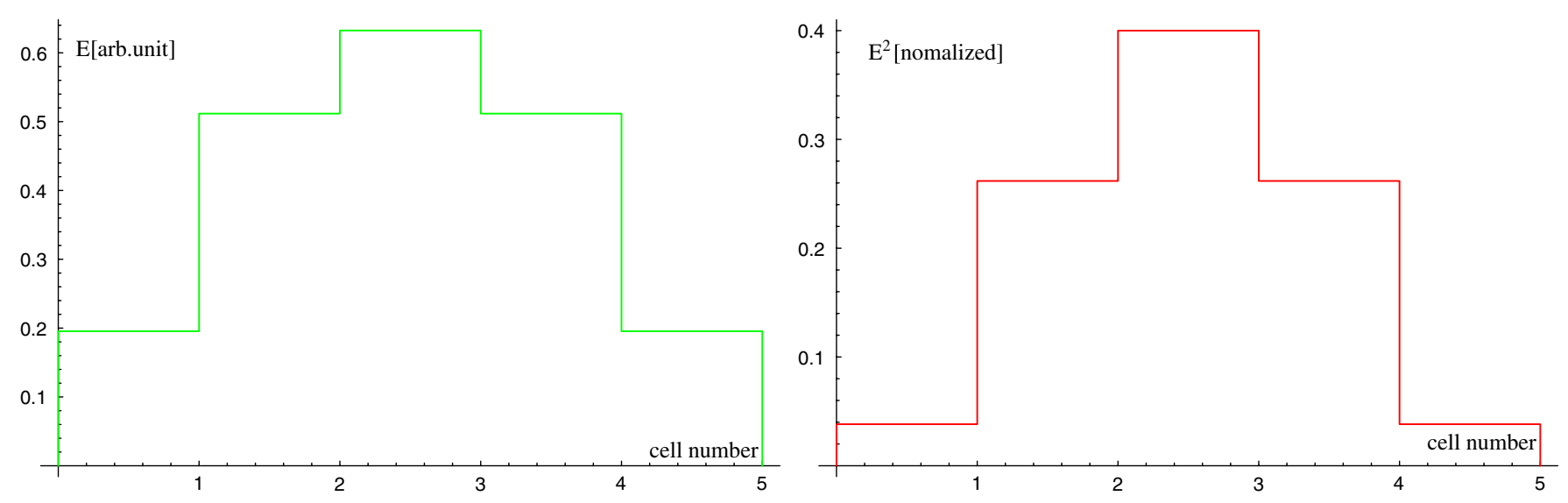

FIG. 12. (Color) Five-cell cavity; left: field profile, right: field-square profile (normalized).
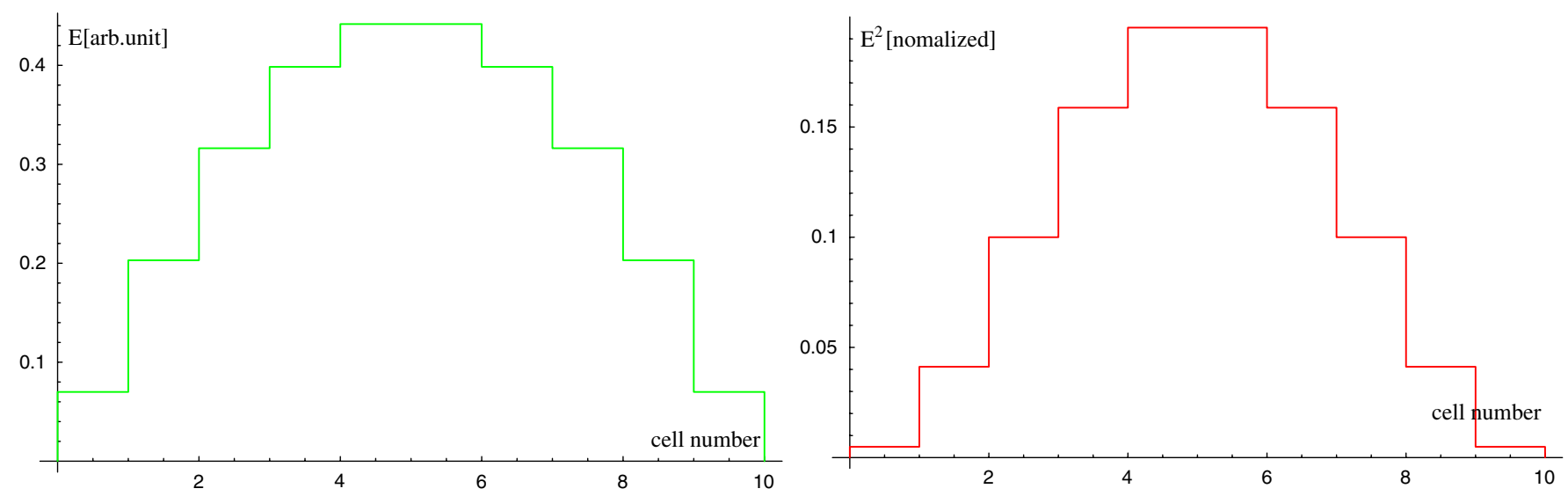

FIG. 13. (Color) Ten-cell cavity; left: field profile, right: field-square profile (normalized). 

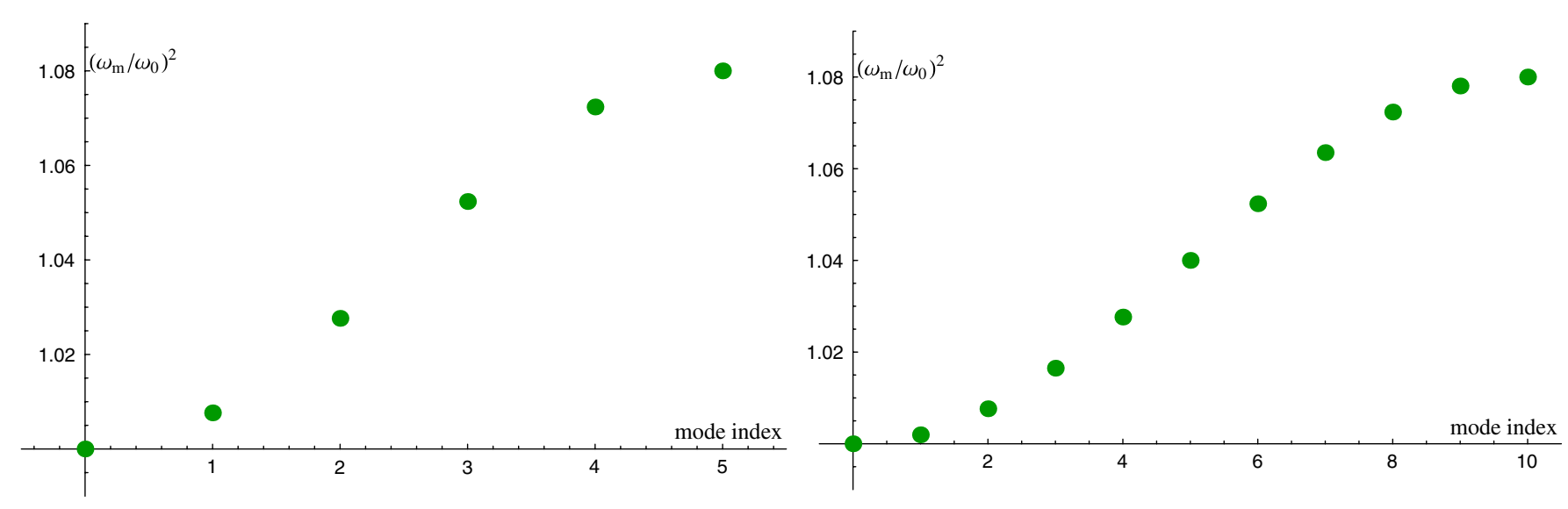

FIG. 14. (Color) Relative mode frequency (squares); left: five-cell, right: ten-cell.

TABLE XIV. HOM parameter scaling for linear frequency scaling and doubling of accelerating cells, which means perfect cells in the worst case, assuming "additive" $(R / Q)$.

\begin{tabular}{lcc}
\hline \hline Parameter & $\begin{array}{c}704.4 \mathrm{MHz}, \\
\text { five-cell }\end{array}$ & $\begin{array}{c}1408.8 \mathrm{MHz}, \\
\text { ten-cell }\end{array}$ \\
\hline$V_{\text {ind }}$ (monopoles) & 1 & $\times 4$ \\
$Z_{\|} / L$ (monopoles) & 1 & $\times 4 \ldots 16$ \\
$\Delta p_{x}$ (dipoles) & 1 & $\times 8 \ldots 32$ \\
$Z_{\perp} / L$ (dipoles, long range) & 1 & $\times 8 \ldots 32$ \\
$I_{\mathrm{BBU}}$ & 1 & $/(8 \ldots 32)$ \\
\hline \hline
\end{tabular}

The impedance as seen by the beam is proportional to $Q_{\text {ext }}$; hence, taking also the disadvantage for the higher frequency into account when simply scaling geometrically the cavity and its ancillaries, one gets the intermediate summary for an exchange of perfect structures of same length: $\left(\boldsymbol{Z}_{\|} / \boldsymbol{L}\right)$ increases by a factor 4 to $16,\left(\boldsymbol{Z}_{\perp} / \boldsymbol{L}\right)$ increases by a factor 8 to 32 , depending on the mode.

The effects of doubling the frequency and number of cells (see Fig. 15) are summarized in Table XIV.

\section{Imperfect structures}

Until now we have not taken into account the necessary end-cell correction and the fabrication tolerances. Because of the presence of the cutoff tubes at the end cells their frequency-even for geometrically perfect cell shape-is different from the center cells which leads-if uncorrected-to an undesired nonflatness of the accelerating mode [40]; the same is true for all HOMs. To get an efficient flat accelerating mode the end-cell shape is geometrically corrected in the cavity design. This geometrical correction is tailored for the field pattern of the accelerating mode and might help for some HOMs but will make things worse for other HOMs.

A second problem-concerning all cells-arises from the fabrication tolerances. Modern SC cavities in the con- sidered frequency range are made from sheet material by spinning, possibly deep drawing for very large series. The resulting product will have a small geometrical scatter that shows up as scatter in the "single-cell frequency." Therefore each fabricated cavity has to be slightly deformed by inelastic detuning to get a reasonably flat field profile and the correct accelerating mode frequency. As for the end-cell correction, the cell detuning is done for the accelerating mode and its action may help or make things worse for the different HOMs but it is extremely improbable that a HOM gets as flat as the targeted accelerating mode.

In this context, one should keep in mind the following points. In theory there is an infinite number of modes, which is in practice limited to the ones that cannot "escape" along the cutoff tubes. For stability considerations, one has to look not at the average over all modes, but rather at the sum, because already a single bad mode is sufficient to significantly limit the machine current. These "bad" modes in general do not couple very well to the HOM couplers close to the end cells but in the laboratory also the reference antennas are on/in the cutoff tube. This means that these bad modes can stay well hidden in the "forest" of spectrum lines, while the visible lines correspond to modes that are well damped. Also modes with high or low $(R / Q)$ cannot be distinguished with this type of measurement. The only way might be a bead-pull measurement but this is very difficult except for the lowest modes that can be well isolated in the forest of lines, which are mostly overlapping in the warm cavity.

The influence of (small) cell frequency changes has been analyzed in [37]. The resulting mathematical problem is identical to the quantum mechanical $(\mathrm{QM})$ perturbation problem; hence, the well-established methods developed there (and the notation) can be applied directly. If one defines a "perturbation operator " (matrix) $P$ [41] with the frequency deviation of cell $k$ from the ideal cell frequency $\delta \omega_{k}=\omega_{k}-\omega_{0}$ as 
TABLE XV. Sensitivity for bad field profiles (lower HOM damping) concerning manufacturing tolerances.

\begin{tabular}{lcc}
\hline \hline Parameter & $\begin{array}{c}704.4 \mathrm{MHz}, \\
\text { five-cell }\end{array}$ & $\begin{array}{c}1408.8 \mathrm{MHz}, \\
\text { ten-cell }\end{array}$ \\
\hline Mode mixing parameter & 1 & $\times 2 \ldots 4$ \\
\hline \hline
\end{tabular}

$$
P=2 \cdot\left(\begin{array}{ccc}
\delta \omega_{1} / \omega_{0} & \cdots & 0 \\
\vdots & \ddots & \vdots \\
0 & \cdots & \delta \omega_{N} / \omega_{0}
\end{array}\right)
$$

one can-in first order-express the perturbed new eigenvectors as a linear combination of the initial unperturbed eigenvectors. The eigenvector changes can be expressed (in QM notation) as

$$
\left|\delta A^{(m)}\right\rangle=\sum_{k \neq m}^{N} \frac{\left\langle A^{(k)}|P| A^{m}\right\rangle}{\left\langle A^{(m)} \mid A^{(k)}\right\rangle} \cdot \frac{\omega_{m}}{2\left(\omega_{m}-\omega_{k}\right)}\left|A^{(k)}\right\rangle .
$$

Without entering into any details, the worrying factor carries the mode frequency difference $\left(\omega_{m}-\omega_{k}\right)$ in the denominator, hence "close" modes are very sensitive to mixing with only small perturbations and this mixing (i.e. adding) results in even more "crooked" field profiles. As an example, in the LEP2 four-cell cavity there is the $\mathrm{TM}_{012}$ mode family [with non-negligible $(R / Q)$, and relatively small coupling factor $K$ ] that is one of the generally about well behaving "lower" modes. When measuring the first $\mathrm{Cu}$ model cavity, "expecting" a perfect field profile for a perfect cavity, we found that the $3 \pi / 4$ mode and the $\pi$ mode were completely mixed resulting in one mode that was mainly excited in one end cell, weakly in the center cell, and nearly not in the opposite end cell while the other mode had the same behavior in opposite sense. This fact was one reason to equip the cavities with one HOM coupler on each side; these couplers were azimuthally not on the same angle to also capture noncircular modes (dipoles, quadrupoles, ...) with different polarization at least at one end. Of course a dipole mode with a similar behavior as the $\mathrm{TM}_{012}$ might have escaped coupling; but LEP2 worked well, so we got away with the chosen design.

If one looks now at the difference of the mode frequencies (Fig. 14), it is evident that the frequency difference between neighboring modes is half for twice the cell number. But at the ends of the passbands the frequency distribution behaves about quadratic, i.e., the two last modes approach by about a factor 4 for the double cell number. As a conclusion of this, we can say that the sensitivity to field profile perturbation increases by a factor 2 to 4.

Very instructive calculated field images for cavities, concerning the end-cell corrections, can be found in [42].

In summary one can give a relative sensitivity for bad field profiles (see Table XV).

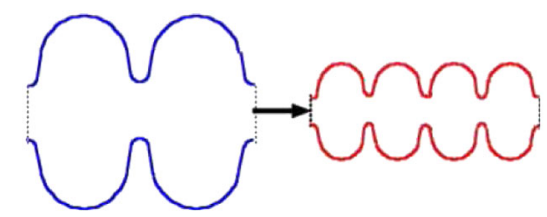

FIG. 15. (Color) Doubling of frequency and doubling of cells.

\section{E. Fast vector feedback}

The SPL cavities have to be equipped with a fast $\mathrm{rf}$ vector feedback. To avoid cross talk, the reference probe and the power coupler are situated on different ends of the cavity. Now the relative polarity of the end cells for modes along the passband (including the accelerating modes passband) flips sign from mode to mode. Therefore, once the feedback is set up for the chosen accelerating mode (generally the $\pi$ mode), the modes with opposite polarity across the cavity (each second one) might start to autooscillate. To avoid this, a complex filter network has to be introduced requiring regular checking of the settings (e.g. each shut-down). There have been a few LEP2 SC cavities in the SPS where the cavities had to be made "invisible" for the proton beam cycle in the SPS. This was done by such a feedback system but-even for "only" four-cell cavities - the two top modes could not be separated by standard filter means. The solution was to use a cable that had $n$ wavelengths for the $\pi$ mode and $n-1 / 2$ wavelengths for the $3 \pi / 4$ mode, introducing a factor -1 in this way.

Evidently when doubling the number of cells and with it the number of modes per passband, the filter system needs more filters but also the mode frequencies get closer-up to a factor 4-making filtering with sufficient attenuation more difficult.

\section{F. Cavity loading in pulsed mode}

In pulsed mode cavities have to be filled with rf energy before beam arrival. During the beam pulse passage the field has to be held up until the last bunch and then this energy is unloaded into an rf load. Therefore this loaded energy is lost, reducing the machine efficiency. Comparing the two cases, the $(R / Q)$ of the higher frequency cavity is twice the one of the lower frequency (see Fig. 16). Therefore the optimum power coupler setting

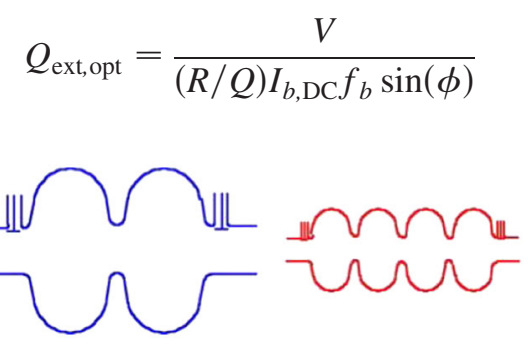

FIG. 16. (Color) Doubling of frequency and doubling of cells: cavity loading. 
is half and the filling time

$$
\tau_{\text {fill }}=Q_{\text {ext }} / \omega
$$

is $1 / 4$ for the higher frequency. The pumping power is given by

$$
P_{\text {pump }}=\frac{V_{\text {equil }}^{2}}{(R / Q) Q_{\text {ext }}}
$$

and is the same for both cases, hence the wasted energy

$$
U_{\text {fill,waste }}=\int_{0}^{\eta \tau_{\text {fill }}} P_{\text {pump }} d t
$$

scales as the filling times, hence the higher frequency has only $1 / 4$ of the wasted rf energy, an advantage for the higher frequency.

\section{G. Transient beam loading}

For injection into subsequent synchrotrons, such as the PS2 or an accumulator ring for neutrino applications, it is foreseen to chop the beam at low energy in order to avoid capture losses during the injection process. For $n_{b}$ bunches, the drop in accelerating voltage during the pulse can be approximated by

$$
\Delta V=\frac{1}{2} T \Delta I \omega\left(\frac{R}{Q}\right)
$$

where $\Delta I$ is the difference between bunch current and average pulse current and $T$ the length of the pulse. For all scenarios, which are considered at the moment the voltage drop was always below 0.5 per mil with respect to the accelerating voltage. This means that for both frequencies transient beam loading due to beam chopping should not be an issue.

\section{H. Conclusions}

Concerning threshold beam current for beam breakup, there is a clear advantage for lower frequencies with a lower number of cells. However, due to the large number of HOMs it is not possible to draw an absolute conclusion, only a risk-gain analysis is possible here. However, one should keep in mind that, in contrast to the usual statistical analysis, where the "average" is important, here the "worst case" is the decisive factor. For an absolute analysis a definitive design has to be tested extensively in simulations as done, e.g., by [43] for SNS.

The energy wasted for cavity loading is in favor (factor 4) of the higher frequency while the rf vector feedback is easier for the lower frequency with less cells.

\section{CRYOGENICS AND CRYOMODULES}

\section{A. Heat loads and required cryogenic capacities}

The same methodology that has been used in the 2006 design report [3] has now been used to estimate the heat loads for the 704.4 and $1408.8 \mathrm{MHz}$ options. We use the newly calculated cavity parameters from Table XII, and the layouts for 704.4 and $1408.8 \mathrm{MHz}$, which are quoted in Table IV. The loads are calculated for the following duty cycles: (1) LP-SPL: the maximum ratings from Table II: $4 \mathrm{GeV}, 1.2 \mathrm{~ms}, 20 \mathrm{~mA}, 2 \mathrm{~Hz} \Rightarrow$ cryoduty cycle of $0.41 \% / 0.28 \%$ (including the filling and decay time in the SC cavities at $704.4 / 1408.8 \mathrm{MHz}$ ); (2) HP-SPL "neutrino": the ratings for neutrino operation from Table III: $5 \mathrm{GeV}, 0.4 \mathrm{~ms}, 40 \mathrm{~mA}, 50 \mathrm{~Hz} \Rightarrow$ cryoduty cycle of $\approx 4.1 \% / 2.5 \%$ (including the filling and decay time in the SC cavities at $704.4 / 1408.8 \mathrm{MHz}$ ). The "cryoduty cycles" are different for the different frequencies because of the different filling (and decay) time of the structures (see Sec. VF).

The definition of the ultimate operation follows the one given in [3] and includes a 10\% increase of the nominal gradient accompanied by a 50\% drop in $Q_{0}$. This approach may be revisited at a later stage but for consistency with the last conceptual design report [3] we keep the same principle.

Focusing on the scenario with the highest loads (HPSPL, $\beta=1$ modules, ultimate operation), the total heat load per cavity at $2 \mathrm{~K}$ is $25 \mathrm{~W}$ (or $14 \mathrm{~W} / \mathrm{m}$ on cryomodule length), which is comparable with the numbers in [3]. For comparison with the values of ILC, reported in the 2007 ILC Reference Design Report [44], the highest heat loads of the HP-SPL cavities are about 20 times those of the ILC cavities. It should be underlined that the ILC report does not refer to an ultimate operation, and that this scenario more than doubles the cavity heat loads in SPL.

Converting total heat loads into installed cryogenic capacity is done by using the formula

$$
\begin{aligned}
Q_{\text {inst }}= & \max \left\{1.5 \cdot\left(1.5 \cdot Q_{\text {static }}+Q_{\text {dyn,nom }}\right) ;\right. \\
& \left.1 \cdot\left(1.5 \cdot Q_{\text {static }}+Q_{\text {dyn,ult }}\right)\right\}
\end{aligned}
$$

which is applied at each temperature level. The resulting 4.5 K equivalent installed capacity for the HP-SPL operating at $2 \mathrm{~K}$ is $20.8 / 18.3 \mathrm{~kW}$, which is $32 \% / 16 \%$ higher than in the 2006 report [3], but still remains in the range of existing state-of-the-art cryogenic plants. However, the meaningfulness of an ultimate mode, which more than doubles the heat loads, needs to be revised.

\section{Operating cavities at $2 \mathrm{~K}$ or $4.5 \mathrm{~K}$}

Assuming the cavity parameters in Table XII, we find that operating the cavities at $4.5 \mathrm{~K}$ would decrease the quality factor of the cavities, and therefore increase the dynamic heat load by a factor of $21(704.4 \mathrm{MHz})$ to 26 $(1408.8 \mathrm{MHz})$. Calculating the required heat capacities for the scenarios listed above yields the values in Tables XVI and XVII for LP- and HP-SPL.

The required installed capacities for LP-SPL remain within reasonable values for $4.5 \mathrm{~K}$ but for the HP-SPL even when running at high duty cycle, it is immediately clear that $4.5 \mathrm{~K}$ is not an option, because it would require 
TABLE XVI. Cryogenic plants for 704.4 MHz, considering a 4 GeV LP-SPL and a 5 GeV HP-SPL.

\begin{tabular}{lccc}
\hline \hline Machine & Temperature $[\mathrm{K}]$ & $Q_{\text {equivalent }}$ at $4.5 \mathrm{~K}[\mathrm{~kW}]$ & $P_{\text {el }}[\mathrm{MW}]$ \\
\hline LP-SPL & 2 & 6.3 & 1.6 \\
LP-SPL & 4.5 & 10.5 & 2.6 \\
HP-SPL neutrino operation & 2 & 20.8 & 5.2 \\
HP-SPL neutrino operation & 4.5 & 95.4 & 23.9 \\
\hline \hline
\end{tabular}

TABLE XVII. Cryogenic plants for $1408.8 \mathrm{MHz}$, considering a 4 GeV LP-SPL and a $5 \mathrm{GeV}$ HP-SPL.

\begin{tabular}{lccc}
\hline \hline Machine & Temperature $[\mathrm{K}]$ & $Q_{\text {equivalent }}, 4.5 \mathrm{k}[\mathrm{kW}]$ & 7.2 \\
\hline LP-SPL & 2 & 10.8 & 1.8 \\
LP-SPL & 4.5 & 18.3 & 2.7 \\
HP-SPL neutrino operation & 2 & 81.9 & 4.6 \\
HP-SPL neutrino operation & 4.5 & 20.5 \\
\hline \hline
\end{tabular}

multiple large cryo plants $(2 \times 25 \mathrm{~kW}$ and above $)$ and becomes unrealistic in terms of space and utilities demand (electricity, cooling water).

Operating at $4.5 \mathrm{~K}$ in saturated pool boiling would offer the advantage of being at a higher pressure than atmospheric (1.3 bars), thus limiting helium contamination due to air leaks. But operating in the boiling regime requires venting of helium vapor bubbles from the uppermost point of all closed envelopes. This would require a specific design of helium vessels, considerably different from ILC/XFEL designs.

\section{B. Cryogenic layout and cooling schemes}

The general cryogenic layout already presented in the 2006 SPL report [3] (Fig. 17) remains a valid option. It consists of three cryogenic strings, one for the low beta cavities (seven cryomodules for an approximate length of $90 \mathrm{~m}$ ) and two for high beta cavities ( 25 cryomodules each, for an approximate length of $380 \mathrm{~m}$ ). Series architecture, similarly to what is proposed for ILC for each cryogenic unit, is used in the cooling scheme, fed by one cryogenics plant placed at one extremity of the SPL. The distribution of cryogen to and back from the cryomodules is ensured by cryogenic lines integrated in the one same cryostat. Cooling of the thermal shielding and heat intercepts is achieved by two levels of gas cooling, at $50-75 \mathrm{~K}$ and at 5-9 K (these temperature ranges may be refined depending on the design of the cryogenic plant and cryomodules). Each string will be individually controlled. A subcooled helium line supplies helium to each string through a JouleThomson (JT) valve to a two-phase (liquid/vapor) line, which ensures cooling of the cavities by a connection at every helium vessel. Phase separators, equipped with helium level gauges and heaters for burning liquid in excess, together with the JT valves and temperature gauges, are housed at the extremities of each string in specific technical service modules. An additional helium feeding line, running along a string and connected to every helium vessel, is used for cooldown/warm-up purposes. At each extremity of the cryomodule, in the interconnection region, the biphase line is connected to a large diameter gas return pipe which runs along the three strings and feeds the gas back to the cold compressors of the cryogenic plant (Fig. 17).

\section{Slope and control issues}

Cooling in the presence of a slope (1.7\%) in the SPL tunnel will certainly have some implications on the liquid helium level control and flow pattern in the two-phase line and possibly lead to limitations on the cooling efficiency of the cavities. These issues certainly need dedicated studies, but are not considered to be show stoppers. It should be remembered that the laser-straight option of ILC would have to cope with similar issues (though with a slope of $0.6 \%$ ) and Fig. 18 illustrates a way to avoid clogging of the two-phase line by a minimum quantity of liquid helium, which drips along the tube and fills each helium vessel. In case cooling limitations are found to be critical, alternative cooling schemes, on reduced string lengths, could be envisaged, but with the drawback of requiring more control equipment (helium filling, phase separators, level, and temperature controls).

\section{Cryomodule design issues}

Preliminary sizing of the gas return pipe indicates that all scenarios of the HP-SPL require inner diameters of $250 \mathrm{~mm}$ or more in order to pump the large mass flows of vapors with a limited pressure difference along the entire length $(\approx 500 \mathrm{~m})$ of the machine.

Therefore, the design solution of the ILC and XFEL cryomodules, where the gas return pipe serves as a structural element for the supporting and aligning of the string of cavities, should certainly be considered. A preliminary cross sectional study, integrating the larger helium vessel of 704.4 MHz cavities into an ILC/XFEL cryostat, shows that the same size of vacuum vessel could be used, but that the thermal shields and supporting structures will have to 

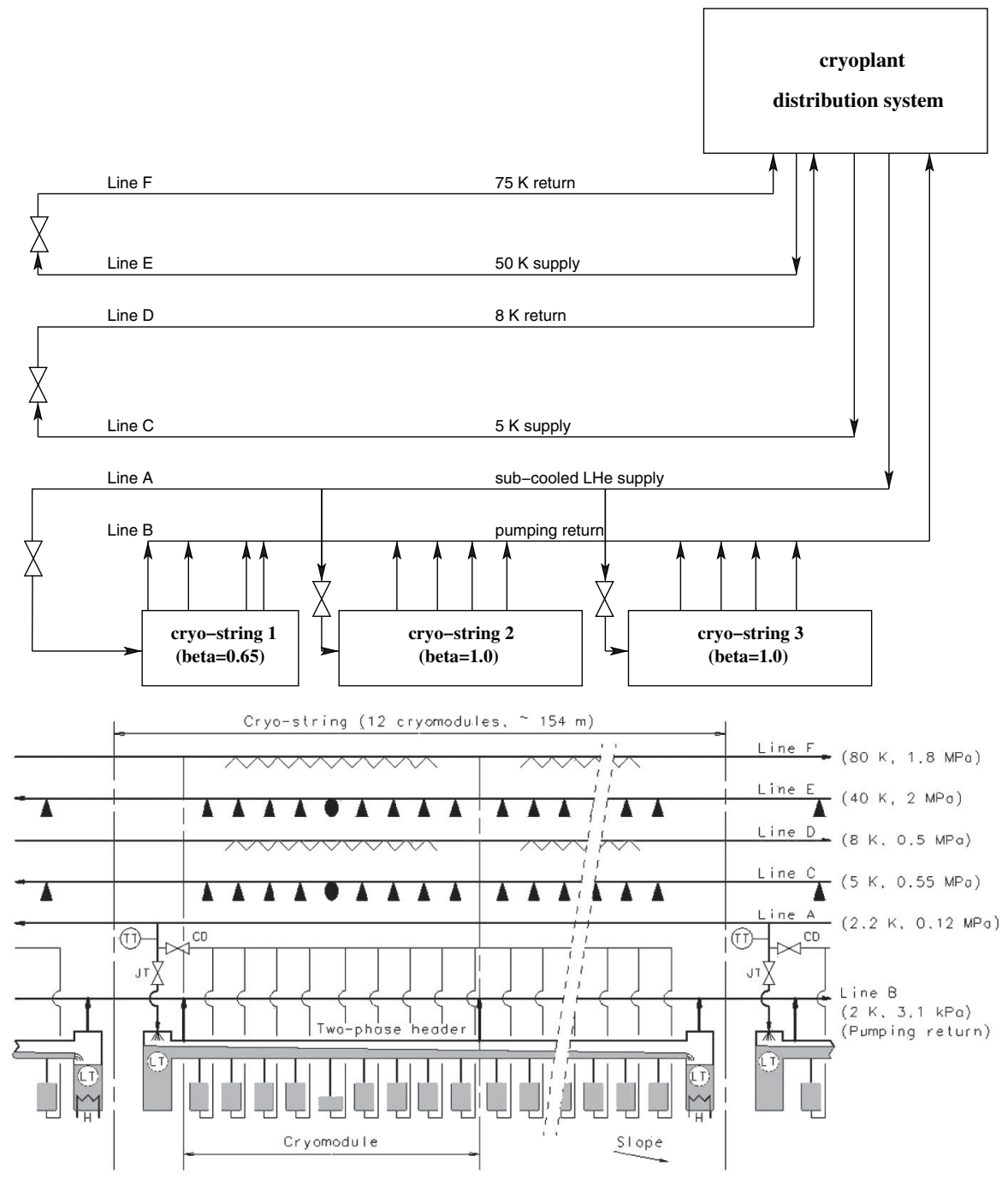

$$
\begin{aligned}
& \begin{array}{c}
\text { Coupler \& Adsarber } \\
\text { heat intercepts }
\end{array} \begin{array}{c}
\text { Current leod } \\
\text { heot intercepts }
\end{array} \quad \square \text { cell cavity } \square \text { Ouadrupole }
\end{aligned}
$$

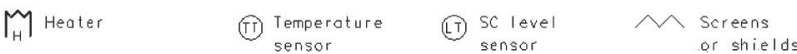

FIG. 17. Possible cooling scheme (published in [44]).

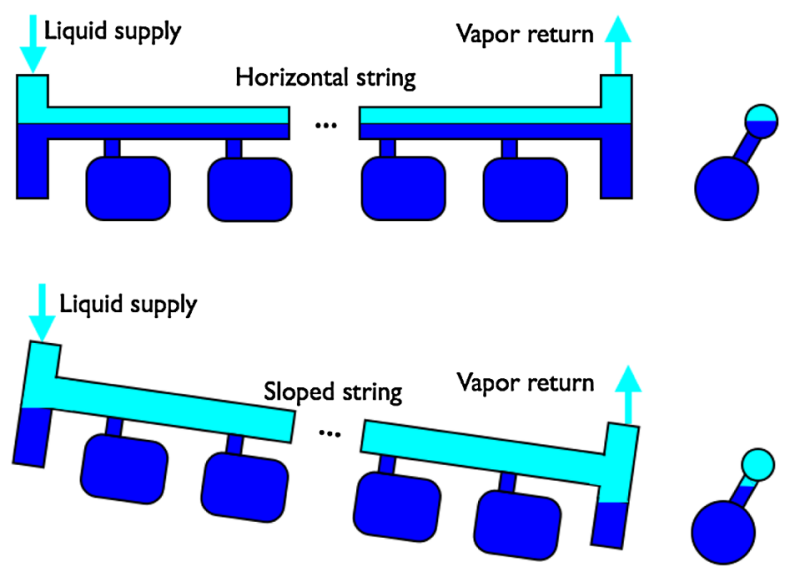

FIG. 18. (Color) Managing two-phase cooling in slopes (published in [44]).

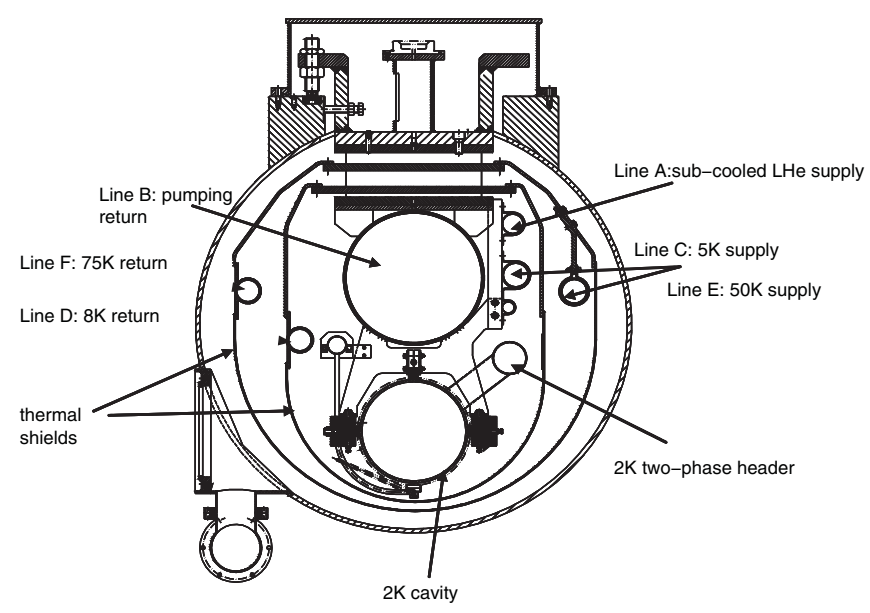

FIG. 19. ILC cryomodule. 
be redesigned (Fig. 19). At this stage of the SPL study, however, it is felt that the design of the cryomodules should not be constrained to fit the vessel of the XFEL cryomodules (in particular for the design restrictions in the longitudinal spacing and sizes of ports), despite the cost saving that making use of existing industrial production lines would bring.

\section{Summary on cryogenics}

A preliminary analysis of the cryogenic infrastructure yields the following conclusions: (i) For the HP-SPL, operation at $4.5 \mathrm{~K}$ would lead to an unreasonably large cryogenic plant, and a power consumption for the cryogenic system that comes close to that of the rf system. (ii) For the LP-SPL the difference between 2 and $4.5 \mathrm{~K}$ operation is not as big as for the HP-SPL, since the system is more dominated by the static losses. This means that one could operate at both temperatures, even though it is clear that a $4.5 \mathrm{~K}$ system would need an installed capacity, which is around $50 \%$ higher than at $2 \mathrm{~K}$. (iii) For both temperatures and frequencies, the cryomodules will have to be adapted for the slope of the SPL (1.7\%). The R\&D effort for either solution is considered comparable. (iv) It seems possible to fit a 704.4 MHz helium vessel into an ILC-sized cryomodule. (v) The heat load in a SPL cryomodule is approximately $20 \times$ higher than in an ILC module. (vi) The SPL cryomodule will reuse many features of the ILC cryomodule. However, a one-to-one copy can certainly not be used. It seems easier to adapt the ILC cryomodule for $1408.8 \mathrm{MHz}$ than for $704.4 \mathrm{MHz}$ but it is unlikely that this will results in major savings.

\section{RF HARDWARE CONSIDERATIONS}

For LP-SPL applications (Table II), the peak forward per cavity is around $500 \mathrm{~kW}$, for both frequencies, with very low average power. The power for the cavities in the low- $\beta$ sections is about half. In the case of SPL for high-power operation (Table III), the peak forward power per cavity rises to up to $1 \mathrm{MW}$ per cavity, with $75 \mathrm{~kW}$ average. The values in Tables XVIII and XIX correspond to optimum coupling for respective current (20 mA or $40 \mathrm{~mA}$ ).

The following analysis compares for both frequencies the availability, development time, size, and cost of klystrons, modulators, cavity power couplers, waveguide system components such as circulators, and loads.

\section{A. Klystrons}

"Off the shelf" devices presently do not exist at either frequency, 704.4 MHz or at 1408.8 MHz. However, the existing klystrons used for SNS, (5 MW peak at $805 \mathrm{MHz}$ ), and for ILC (5 MW and $10 \mathrm{MW}, 1.3 \mathrm{GHz}$ ) are sufficiently close in frequency to be used as references. However, the duty cycles in these machines are somewhat different from the SPL values.

The 5 MW SNS klystron operating at $1.5 \mathrm{~ms} / 60 \mathrm{~Hz}$ duty cycle and $450 \mathrm{~kW}$ average power seems at the limit of the state of the art, but this power is nevertheless reachable at 704.4 MHz. On paper, a 704.4 MHz multibeam klystron (MBK) operating at even higher power looks very attractive. Its feasibility however, is a completely different question. Hence, we will base our 704.4 MHz configuration on $5 \mathrm{MW}$ klystrons, one klystron powering eight cavities in the LP-SPL, and one klystron powering four cavities in the high- $\beta$ section of SPL, with an average power of up to $300 \mathrm{~kW}$.

For $1408.8 \mathrm{MHz}$ we can consider a $5 \mathrm{MW}$ klystron feeding eight cavities in LP-SPL, with either the same 5 MW klystron feeding four cavities in SPL, or a $10 \mathrm{MW}$ klystron feeding eight cavities. The latter needs $500 \mathrm{~kW}$ average power. However, reduced collector dimensions when scaling to a higher frequency impose limits on average power. Upgrading the existing ILC tubes, in particular, the $10 \mathrm{MW}$ MBKs to $50 \mathrm{~Hz}$ repetition rate while keeping the pulse duration at $1.5 \mathrm{~ms}$, would need substantial R\&D.

TABLE XVIII. Maximum rf power per cavity when operating at $704.4 \mathrm{MHz}$.

\begin{tabular}{lccccr}
\hline \hline Operation at 704.4 MHz & \multicolumn{2}{c}{$P_{\text {peak }}[\mathrm{kW}]$} & \multicolumn{2}{c}{$P_{\text {av }}[\mathrm{kW}]$} \\
Cavity $\beta$ & rf duty cycle [\%] & 0.65 & 0.92 & 0.65 & 0.92 \\
\hline LP-SPL & 0.39 & 270 & 475 & 1.0 & 1.8 \\
HP-SPL neutrino operation (0.4 ms) & 3.92 & 540 & 950 & 21 & 37 \\
HP-SPL "high-power EURISOL (1.2 ms)" & 7.92 & 540 & 950 & 42 & 75 \\
\hline \hline
\end{tabular}

TABLE XIX. Maximum rf power per cavity when operating at $1408.8 \mathrm{MHz}$.

\begin{tabular}{|c|c|c|c|c|c|c|c|}
\hline \multirow{2}{*}{$\begin{array}{l}\text { Operation at } 1408.8 \mathrm{MHz} \\
\text { Cavity } \beta\end{array}$} & \multirow{2}{*}{ rf duty cycle $[\%]$} & \multicolumn{3}{|c|}{$P_{\text {peak }}[\mathrm{kW}]$} & \multicolumn{3}{|c|}{$P_{\mathrm{av}}[\mathrm{kW}]$} \\
\hline & & 0.6 & 0.76 & 0.94 & 0.6 & 0.76 & 0.94 \\
\hline LP-SPL & 0.28 & 360 & 435 & 490 & 1.0 & 1.2 & 1.4 \\
\hline HP-SPL neutrino operation $(0.4 \mathrm{~ms})$ & 2.48 & 720 & 870 & 980 & 18 & 22 & 24 \\
\hline HP-SPL "high-power EURISOL (1.2 ms)" & 6.48 & 720 & 870 & 980 & 47 & 56 & 63 \\
\hline
\end{tabular}


TABLE XX. Summary of statements concerning the frequency choice (disadvantages in italic, advantages in upright font, decisive criteria in bold).

\begin{tabular}{|c|c|}
\hline Subject & $1408.8 \mathrm{MHz}$ \\
\hline General accelerator issues & $\begin{array}{l}\text { Lower efficiency } \\
\text { (beam power/grid power) due } \\
\text { to the larger stored energy } \\
\text { in the cavities. } \\
\text { Only two different cavity types. }\end{array}$ \\
\hline Beam dynamics/HOM & $\begin{array}{l}\text { Higher longitudinal emittance growth. } \\
\text { Significantly higher risk for trapped modes, } \\
\text { beam breakup, and other collective effects. } \\
\text { Higher sensitivity to longitudinal } \\
\text { beam errors from Linac4 }\end{array}$ \\
\hline SC cavities & $\begin{array}{l}\text { Gradient depends only on geometry (optimum } \beta \text { ), not on frequency. } \\
\text { Usable gradient (with acceptable yield) is likely to be lower than presently foreseen: } \\
\text { to be studied. } \\
\text { Control of mechanical vibrations is expected to be of a similar level of difficulty, } \\
\text { thanks to the girder. }\end{array}$ \\
\hline rf equipment & $\begin{array}{l}\text { Klystrons difficult to build } \\
\text { (average power density limitations). } \\
\text { Extremely challenging specifications for } \\
\text { the design of most high-power rf components. } \\
\text { Most of these components will have to be } \\
\text { increased in volume/complexity to deal } \\
\text { with the high average power densities. }\end{array}$ \\
\hline Cryogenics & $\begin{array}{l}\text { Similar cooling needs. } \\
\text { Possibility to reuse some of the ILC cryomodule engineering design in both cases. }\end{array}$ \\
\hline Other issues & $\begin{array}{l}\text { Availability of SUPRATECH test } \\
\text { place in France. } \\
\text { Possible synergy with ESS. }\end{array}$ \\
\hline
\end{tabular}

Obviously the collector would need to be redesigned. There are many other uncertainties such as cavity layout, internal heating of certain areas inside the tube, etc. XFEL MBKs are intended to be operated up to $30 \mathrm{~Hz}$, but at reduced pulse duration or amplitude in order to keep the beam power in the tube below $250 \mathrm{~kW}$ (i.e. only about $50 \%$ of the SPL needs).

\section{B. Modulators}

For the modulator ratings, the frequency, whether it is for $704.4 \mathrm{MHz}$ or $1408.8 \mathrm{MHz}$, makes no fundamental difference. The peak power and repetition rate are the most important factors. The modulator for $50 \mathrm{~Hz}$ operation is an extremely difficult challenge, but it is outside the scope of this review.

\section{Power distribution}

There is probably no difficulty to operate either WR1150 or WR650 waveguides at MW, with $1.5 \mathrm{~ms}$ and $50 \mathrm{~Hz}$. For 704.4 MHz WR1150 waveguides are preferred, and for $1408.8 \mathrm{MHz}$ smaller WR650 may be used. Smaller waveguides have the advantage of reduced size and larger ones the advantage of lower power losses. Both systems would require the use of SF6 gas.

However, for components like circulators, rf loads, power splitters, phase shifters, and vector modulators the power dissipation becomes the limiting factor. Again the question of heating must be addressed and obviously the smaller the components are the more important the problem is. Hence, in a $1408.8 \mathrm{MHz}$ high-power system, although the components will be more compact due to the waveguide size, doubling up of certain critical components may be required, with considerably increased complexity and an overall increase in required space, compared to an equivalent 704.4 MHz system.

SNS $805 \mathrm{MHz}$ circulators and rf loads comply with SPL duty cycles and average powers. On the other hand, the new and sophisticated WR650 waveguide components developed for TTF, ILC, and XFEL, which represent the state of the art in the domain of waveguide components, can only handle 4 to $5 \mathrm{~kW}$ average power while much higher levels are needed for SPL. 


\section{Cavity power couplers}

In SPL the power constraints, $1 \mathrm{MW}$ peak power and up to $75 \mathrm{~kW}$ average power per cavity, are also challenging. The 704.4 MHz 1 MW power coupler developed at CEA within HIPPI is due to be tested soon. Recent information indicates that further development will continue in the frame of FP7. At $1.3 \mathrm{GHz}$, TTF and XFEL couplers performances, $280 \mathrm{~kW}$ and $10 \mathrm{~Hz}$, are far from the SPL target values. However, there are nevertheless some very interesting high frequency coupler developments in progress, with indications, for example, that modified TTF couplers at Cornell have reached $61 \mathrm{~kW}$ average power and $1.3 \mathrm{GHz}$ couplers (ERL) have been tested up to $50 \mathrm{~kW} \mathrm{cw}$. Further intensive development followed by extensive testing is required whatever the frequency choice. Variable couplers, an interesting option if an upgrade from LP-SPL to full SPL were to be done, are probably outside the scope of the project. Coupling to the higher current of $40 \mathrm{~mA}$ would mean a relatively low penalty of $11 \%$ in reflected power when running LP-SPL at $20 \mathrm{~mA}$.

\section{E. Summary}

For the rf power system considerations, 1408.8 and 704.4 MHz systems are both completely feasible for LPSPL. Klystrons can be easily available at both frequencies for the LP-SPL. However, the high average power required by the HP-SPL will be very difficult to achieve at 1408.8 MHz (klystrons, power couplers, and waveguide components). A frequency of $704.4 \mathrm{MHz}$ is therefore preferred.

\section{CONCLUSIONS}

\section{A. Temperature}

According to the analysis in the previous sections, power efficiency requires the operating temperature of the superconducting cavities to be in the vicinity of $2 \mathrm{~K}$. This conclusion seems clear for the low-power version of the SPL, and it is mandatory for high power.

\section{B. Frequency}

The use of a frequency close to $1.3 \mathrm{GHz}$ was expected to allow reusing the design and the engineering experience accumulated for the ILC. As a matter of fact, the ILC drawings cannot be reused as such for the SPL because the frequency is not the same (1.4 instead of $1.3 \mathrm{GHz})$ and because the input and HOM couplers have to be different due to the larger instantaneous beam current $(40 \mathrm{~mA}$ instead of $11 \mathrm{~mA})$ and to the higher rf duty factor (4\%-8\% instead of 1\%). Whatever the frequency, extensive use can however be made of the knowledge gained and of the basic design principles adopted in the context of the ILC (helium return pipe used as alignment girder inside the cryomodule, for example).
The other statements concerning the choice of the frequency are summarized in Table IV. The first observation is that the accelerating field in superconducting cavities is quasi-independent of frequency, which results in a longer linac at $1408.8 \mathrm{MHz}$ and in a larger variety of cavities because of their higher number of cells. The main arguments in favor of $1408.8 \mathrm{MHz}$ are that the electrical efficiency is better (less stored energy in the cavities) and the rf components in general are expected to be of smaller size. These are outweighed by the difficulty (impossibility?) to build rf devices (klystrons, waveguide components) capable of the SPL duty factor, which can cope with the required average power densities. Moreover, $704.4 \mathrm{MHz}$ is more tolerant in terms of field stability in Linac4 and it is a possible common choice for future high-power proton accelerators in Europe (ESS, ADS). A high-power 704.4 MHz test place with cryogenic cooling capability already exists in France.

A frequency of 704.4 MHZ is therefore considered as the appropriate frequency to be used in the superconducting part of the SPL.

\section{Gradient}

The accelerating gradients of $19(\beta=0.65)$ and $25 \mathrm{MV} / \mathrm{m}(\beta=1)$ assumed in the conceptual design report of the SPL II [3] seem difficult to achieve with an acceptable production yield, according to the survey reported in Sec. IV of the performance achieved by recently built superconducting cavities. A more realistic figure at $\beta=1$ is in the range $16-23 \mathrm{MV} / \mathrm{m}$. It is important that new SPL prototype cavities operating at $704.4 \mathrm{MHz}$ are built and tested during the next few years to correctly define the gradients to be used in the SPL design.

A summary of all advantages and disadvantages of choosing 704.4 or $1408.8 \mathrm{MHz}$ is presented in Table XX.

[1] R. Garoby and M. Vretenar, PS/RF/Note 96-27.

[2] M. Vretenar, CERN Report No. CERN-2000-012, http:// doc.cern.ch/yellowrep/2000/2000-012/p1.pdf.

[3] F. Gerigk, CERN Report No. CERN-2006-006, http:// doc.cern.ch/yellowrep/2006/2006-006/full_document.pdf.

[4] R. Garoby, CERN Report No. CERN-AB-2007-074 PAF, http://doc.cern.ch/archive/electronic/cern/preprints/ab/ab2007-074.pdf.

[5] R. Garoby and F. Gerigk, CERN Report No. CERN-AB2007-016 RF, http://cdsweb.cern.ch/record/1035211/files/ ab-2007-016.pdf.

[6] M. Benedikt, CERN Report No. CERN 2007-002, p. 117, http://doc.cern.ch//archive/cernrep/2007/2007-002/p117. pdf.

[7] F. Gerigk and M. Vretenar, Linac4 Technical Design Report, CERN-AB-2006-084 ABP/RF, http://doc.cern. ch/archive/electronic/cern/preprints/ab/ab-2006-084.pdf.

[8] Status of SPL rf Frequency and Cooling Temperature, CERN, 2008, http://indico.cern.ch/conferenceDisplay.py? confId $=32631$. 
[9] Eurisol Design Study: http://www.eurisol.org/site01/ index.php.

[10] EURISOL is a proposed European facility for the production of radioactive ion beams (RIB) using a MW-class proton driver.

[11] P. Zucchelli, Phys. Lett. B 532, 166 (2002).

[12] J.E. Campagne and A. Caze, CERN-NUFACT-NOTE 142, 2004, http://cdsweb.cern.ch/record/806547.

[13] The International Design Study for the Neutrino Factory, https://www.ids-nf.org/wiki/FrontPage.

[14] F. Gerigk and R. Garoby, in Proceedings of the 39th ICFA Advanced Beam Dynamics Workshop on High Intensity High Brightness Hadron Beams, 2006 (CERN CERN-AB2006-020, 2006), http://cdsweb.cern.ch/record/961205.

[15] R. Garoby, M. Benedikt, A. Fabich, and F. Gerigk, CERN Report No. CERN-AB-2007-014, http://cdsweb.cern.ch/ record/1029954.

[16] ISOLDE is a CERN based RIB facility, which is supplied with protons by the PSB.

[17] E. Noah et al., EURISOL Target Stations Operation and Implications for its Proton Linac Driver Beam, EPAC06, Edinburgh, http://cdsweb.cern.ch/record/971892.

[18] M. Aiba, CERN Report No. CERN-AB-2008-060, http:// cdsweb.cern.ch/record/1125556.

[19] M. Bonesini, Particle Production vs Energy, NuFact08, Valencia.

[20] The geometrical beta of a cavity refers to its cell length (iris to iris), which is given by $\beta \lambda / 2$, where $\lambda$ refers to the rf wave length.

[21] R. Duperrier, N. Pichoff, and D. Uriot, in Proceedings of the International Conference on Computational Science: ICCS 2002, Amsterdam, The Netherlands, 2002 (SpringerVerlag, Berlin, 2002).

[22] A. Perrin and J. F. Amand, Travel v4.06, User's Manual, CERN (2003).

[23] R. Duperrier, N. Pichoff, and D. Uriot, Phys. Rev. ST Accel. Beams 10, 084201 (2007).

[24] W. Weingarten, CERN Report No. CERN-AB-2008-063, http://cdsweb.cern.ch/record/1132351.

[25] D. C. Mattis and J. Bardeen, Phys. Rev. 111, 412 (1958).

[26] The theoretical magnetic field limit for a perfect superconducting surface is the superheating field $B_{\text {sh }}$, beyond which magnetic flux will enter into the superconductor. Real surfaces of type II superconductors will allow flux entry already at the lower critical field $B_{c 1}<B_{\text {sh }}$. More details may be found in textbooks, e.g. [45].

[27] The thermal conductivity $\lambda$ for niobium at $4.2 \mathrm{~K}$ is related to the residual resistivity ratio (RRR) by $\lambda /(\mathrm{W} /(\mathrm{mK}))=$ $\mathrm{RRR} / 4$.

[28] This equation is strictly speaking only valid for $T<T_{c} / 2$; the factor $A$ depends on several material parameters and the numerical value $A=0.75 \times 10^{5} \mathrm{n} \Omega \mathrm{K} /(\mathrm{GHz})^{2}$ may can be considered as typical for "cavity grade" niobium with a residual resistivity ratio $(\mathrm{RRR})$ of $\approx 100$. The dependence of the energy gap $\Delta$ on the magnetic field and temperature were neglected (cf. [46]). Strictly speaking, the surface resistance is composed of the BCS part and the comparatively smaller residual surface resistance $R_{\text {res }}$ as complement (which is stochastic by its nature).

[29] In addition, due to the relatively large thermal diffusivity of niobium $\lambda /(c \cdot \rho) \approx 9 \times 10^{-3}\left[\mathrm{~m}^{2} / \mathrm{s}\right]$, a sensible temperature increase across a $3 \mathrm{~mm}$ thick cavity wall upon a stepwise rf power increase will take about $1 \mathrm{~ms}$, close to the pulse length of the SPL and, hence, the temperature increase across the cavity wall is even more attenuated.

[30] DESY has demonstrated that the production yield for electropolished cavities is even larger than this number, which was obtained by essentially analyzing the test results of chemically polished cavities; cf. [47].

[31] A. Polyanskii, A. Squitieri, M. Jewell, P. Lee, A. Gurevich, D. Larbalestier, P. Bauer, C. Boffo, L. Bellantoni, and H. Edwards, in the 2005 Workshop on RF Superconductivity, Ithaca, NY.

[32] The magnetically induced surface resistance $R_{s}^{m}$ is usually negligible by providing sufficient magnetic shielding and, hence, will not be considered any further.

[33] C. Benvenuti et al.,Physica (Amsterdam) 316C, 153 (1999).

[34] W. Weingarten, Part. Accel. 53, 199 (1996), http:// cdsweb.cern.ch/record/296351.

[35] Ph. Bernard, E. Chiaveri, and J. Tückmantel, "Technical and Financial Implications of the Frequency Choice for a SC Accelerator Section" (unpublished).

[36] A. Lombardi (private communication).

[37] E. Haebel and J. Tückmantel, CERN Report No. CERN/ EF/RF 81-5, 1981.

[38] Passband refers to the frequency bandwidth of a mode family (see, for instance, the dispersion curve in Fig. 11). For each cell of the cavity one can find one mode on this dispersion curve.

[39] The term cutoff tubes describes the beam tubes, which are attached on either end of the cavity. In many cases these tubes start with a large diameter, which allows the mounting of a HOM or rf coupler. After the coupler section the diameter is reduced, so that the "cutoff" frequency of the beam tube is higher than the frequency of the accelerating mode. This measure prevents the accelerating mode from "travel out of the cavity."

[40] Nonflatness means that the peaks of the electric on-axis electric field are different from cell to cell.

[41] In comparison to [37] we have additionally approximated $\omega_{k}^{2}-\omega_{m}^{2}$ by $2 \omega_{m}\left(\omega_{k}-\omega_{m}\right)$.

[42] J. Sekutowicz, in Proceedings of the Linear Accelerator Conference (LINAC06), Knoxville, 2006.

[43] R. Sundelin, D.-O. Jeon, S.-H. Kim, and M. Doleans, in Proceedings of the Particle Accelerator Conference, Chicago, IL, 2001 (IEEE, New York, 2001), http:// cern.ch/AccelConf/p01/PAPERS/TPPH135.PDF.

[44] International Linear Collider Reference Design Report: Volume 3: Accelerator, edited by N. Phinney, N. Toge, and N. Walker, Reports No. ILC-REPORT-2007-001, No. CERN-2007-006, and No. DESY-FLC, Hamburg, Germany (2007).

[45] H. Padamsee, J. Knobloch, and T. Hays, RF Superconductivity for Accelerators (Wiley-VCH Verlag Weinheim, 2008, ISBN 978-3-527-40842-9).

[46] R. Meservey and B. B. Schwartz, in Superconductivity, edited by R. D. Parks (Marcel Dekker, New York, 1969), Vol. 1, p. 117.

[47] L. Lilje et al., Nucl. Instrum. Methods Phys. Res., Sect. A 524, 1 (2004). 